\title{
THESIS
}

\section{A SURVEY OF THE ARTHROPOD FAUNA ASSOCIATED WITH HEMP (CANNABIS SATIVA L.) GROWN IN EASTERN COLORADO}

\author{
Submitted by \\ Melissa Schreiner \\ Department of Bioagricultural Sciences and Pest Management
}

In partial fulfillment of the requirements

For the Degree of Master of Science

Colorado State University

Fort Collins, Colorado

Fall 2019

Master's Committee:

Advisor: Whitney Cranshaw

Frank Peairs

Mark Uchanski 
Copyright by Melissa Schreiner 2019

All Rights Reserved 


\begin{abstract}
A SURVEY OF THE ARTHROPOD FAUNA ASSOCIATED WITH HEMP (CANNABIS SATIVA L.) GROWN IN EASTERN COLORADO
\end{abstract}

Industrial hemp was found to support a diverse complex of arthropods in the surveys of hemp fields in eastern Colorado. Seventy-three families of arthropods were collected from hemp grown in eight counties in Colorado in 2016, 2017, and 2018. Other important groups found in collections were of the order Diptera, Coleoptera, and Hemiptera. The arthropods present in fields had a range of association with the crop and included herbivores, natural enemies, pollen feeders, and incidental species. Hemp cultivars grown for seed and fiber had higher insect species richness compared to hemp grown for cannabidiol (CBD). This observational field survey of hemp serves as the first checklist of arthropods associated with the crop in eastern Colorado. Emerging key pests of the crop that are described include: corn earworm (Helicoverpa zea (Boddie)), hemp russet mite (Aculops cannibicola (Farkas)), cannabis aphid (Phorodon cannabis (Passerini)), and Eurasian hemp borer (Grapholita delineana (Walker)). Local outbreaks of several species of grasshoppers were observed and produced significant crop injury, particularly twostriped grasshopper (Melanoplus bivittatus (Say)). Approximately half (46\%) of the arthropods collected in sweep net samples during the three year sampling period were categorized as predators, natural enemies of arthropods. These findings are of significance considering the expanding cultivation of hemp both within the region of study and across the United States. A website developed to extend the information developed in this project has been accessed over 20,000 times in 28 months, indicating there is wide interest in this subject area. 


\section{ACKNOWLEDGEMENTS}

I wish to thank my family and friends, for without them I would not have had the support, which I needed to complete this degree. I would like to acknowledge the substantial funding provided by the Western Region IPM Center and the CSU Experiment Station. Thank you to the participating farmers, production farms, and research stations that permitted us to sample arthropods from their facilities across Colorado. Thank you to the Fat Pig Society, Colorado Cultivars, and the Colorado Department of Agriculture for providing resources to conduct local hemp trials, insect collection, and analysis. Thank you to all the lab members: Andrew Miller, Wendlin Burns, Erika Peirce, Rasha Al-akeel, Colton O'Brien, Janet Hardin, Tristan Kubik, Sara Painter, Jacob Pitt, Brian Mitchell, and Tyler Yamashita for their collaboration, technical assistance, and support on various projects. I am indebted to the director of the C.P. Gillette Museum of Arthropod Diversity, Dr. Boris Kondratieff, both for the assistance on identification of our arthropod specimens but also for his longtime mentorship of insect taxonomy at this land grant institution. Thank you to Dr. Rachael Sitz, for her training and her contagious appetite for knowledge. Thank you to my department head, Dr. Amy Charkowski, for encouraging students and aiding the diverse research produced from our multifaceted department. I express tremendous gratitude to my graduate committee members Dr. Frank Peairs and Dr. Mark Uchanski for their continued support, lab space, field space, and cooperative insights on research. With my highest respects I would like to recognize my graduate advisor, Dr. Whitney Crashaw, for providing his endless encouragement, guidance, and entomological education over this degree. 


\section{TABLE OF CONTENTS}

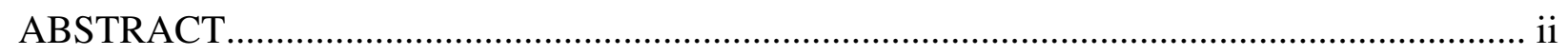

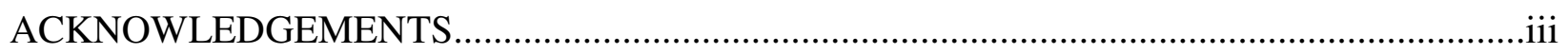

1. CHAPTER 1: INTRODUCTION TO HEMP (CANNABIS SATIVA L).....................................

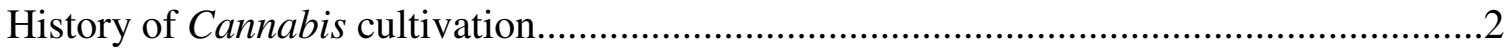

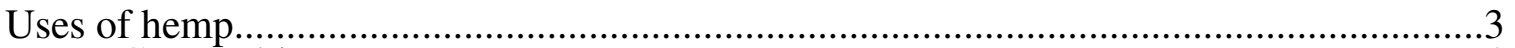

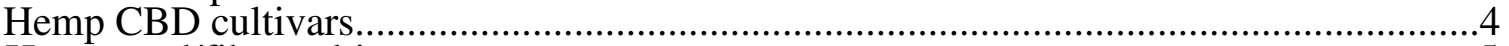

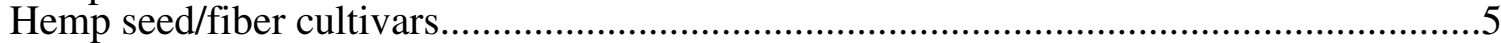

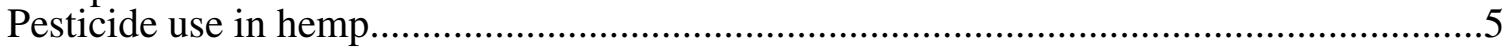

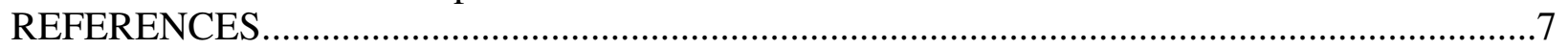

2. CHAPTER 2 - A SURVEY OF THE ARTHROPOD FAUNA ASSOCIATED WITH HEMP

(CANNABIS SATIVA L.) GROWN IN EASTERN COLORADO.............................................10

Materials and methods.........................................................................................

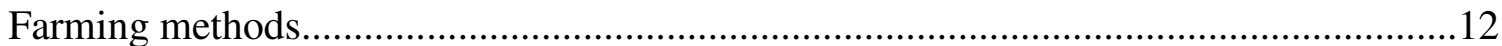

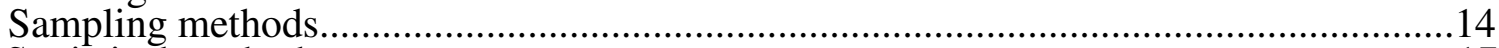

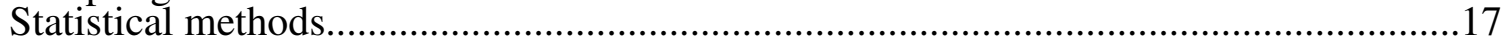

Results and discussion.........................................................................................18

Arthropods found in hemp and their association with crop/feeding guilds...........31

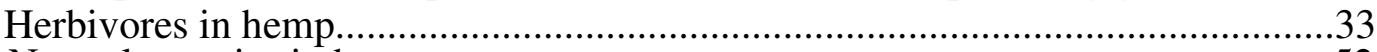

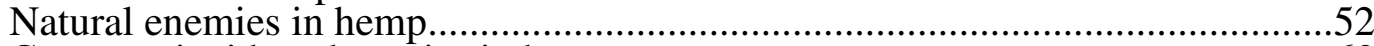

Common incidental species in hemp................................................................60

Effects of hemp cultivar type on arthropod diversity...........................................66

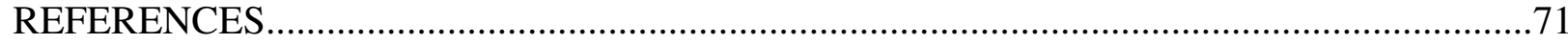

3. CHAPTER 3: THE EFFECT OF INSECTICIDES ON HEMP RUSSET MITES (ACULOPS

CANNIBICOLA) INFESTING HEMP (CANNABIS SATIVA L.).............................................8

Methods and materials............................................................................................... 81

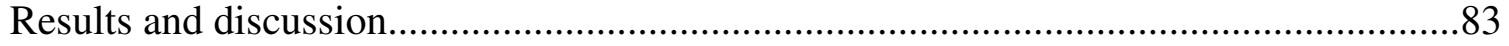

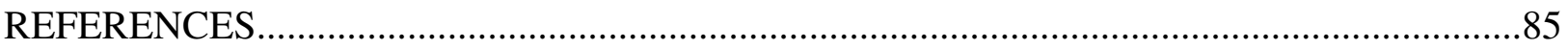

4. CHAPTER 4: MANAGEMENT RESOURCES FOR HEMP FARMERS PROVIDED BY

THE CSU HEMP INSECT WEBSITE.................................................................................

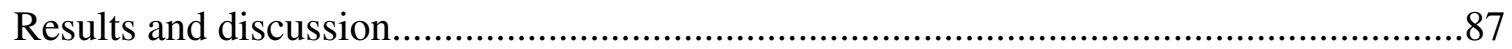

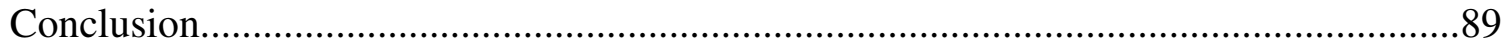

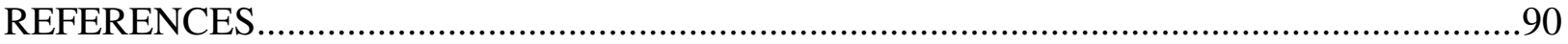




\section{CHAPTER 1 - INTRODUCTION TO CANNABIS SATIVA L.}

Worldwide there are over 30 nations that grow industrial hemp (Cannabis sativa L.) as an agricultural commodity (Johnson, 2014). After a hiatus of about 70 years the United States rejoined the global market when the 2014 Farm Bill was amended to allow states to create legislation for commercial production of hemp; University research on hemp was additionally sanctioned within this legislation. At present 41 states have passed some sort of legislation enabling production of hemp (Cranshaw et al., 2019) along with other industries associated with Cannabis sativa. Colorado has been the lead state in this new era of hemp production, with production growing from 12,042 licensed acres of (346 growers) in 2017 to 30,950 (835 growers) in 2018.

The future of US industrial hemp production will be dependent on many developments including improvements in crop production, breeding and seed quality, development of consumer goods, and the creation of processing facilities. Since the reemergence of hemp as a crop grown in the United States there has been enormous interest that has attracted large numbers of producers. Following clarifying language regarding hemp production in the 2018 Farm Bill, there have been sharp increases in first time producers across the country, although there are voices that suggest the high level of enthusiasm may be difficult to sustain if problems with overproduction and insufficient market development (Cherney and Small 2016) are not addressed. In addition, sustainable production of industrial hemp in the United States will also need support through updated legislation and government regulations, and research into all aspects of its production, including methods to manage pest species of the crop. 


\section{History of hemp cultivation}

Hemp was one of the first fiber crops grown by early agriculturalists (Small, 2015). The first evidence of cultivation was found in the Middle East, c. 800-900 BCE, and Central Asia, between approximately 700 and 300BCE, where hemp was commonly used as a source for fiber (Rull and Vegas-Vilarrúbia, 2014). Hemp has been a versatile crop used across many cultures for over 3000 years $(\mathrm{Li}, 1973)$ to provide such essentials as rope, cloth, and paper.

Hemp was originally introduced to the US in the 1600s as a fiber crop to supply the colonies with rope and textile-based products (Booth, 2005). Pioneers on trails leading west used hemp to make supplies for Conestoga wagons (Deitch, 2003). Betsy Ross made some of the first American flags out of hemp fiber in 1776, and cultivation of hemp for fiber thrived for many decades. In 1942, the USDA initiated the "Hemp for Victory" campaign which asked farmers to plant hemp for fiber to supply the US navy with materials for sailing such as rope and canvas (Willsie, 1942).

Because of federal restrictions directed to suppressing drug forms of Cannabis sativa (e.g., marijuana) US production sharply declined following the Marijuana Tax Act (1937). There was a brief resurgence during World War II following relaxation of this law to enable production for hemp-derived material for the war effort, but production again declined sharply after the war and essentially all legal production ceased with the Controlled Substances Act (1971) that classified the plant as a Class I drug subject to new drug enforcement laws. Since the early 1950s these regulations so affected economics of production that competition from other fibers and building materials (i.e. cotton and timber) completely replaced hemp within the country. Until very recently essentially all other production of this crop has been outside of the United States, notably in Canada and Europe. 


\section{Uses of hemp}

In 1938, Popular Mechanics, a classic magazine of popular science and technology wrote how hemp could be used in 25,000 different products (Anonymous, 1938). Cannabis has been selectively bred for many diverse purposes; one being the extractable cannabinoids used for pharmacological or recreational purposes. For thousands of years, cannabis, has been valued as a versatile and quick producing herbal medicine (Pain 2015; Hill 2015). Hemp provides one of the best of all plant fibers for making textiles and rope. The fibers can be used to create clothing, insulation, or composite material (Nature 1996). Hemp fiber is also used for thousands of products making paper, cardboard, concrete, biodegradable plastics, fuel, and other building materials.

Hemp has great potential as a fiber source for the future as it produces up to four times as much cellulose per acre as timber (Dewey and Merrill 1916). Northern American forests have been heavily logged and up to $70 \%$ of those native forests have been removed (Bowyer 2001). Hemp grows faster than timber and can replace these crops in most applications. Hempcrete is bio-composite constructed from hemp shive, lime binder and water. Hemp bricks and can be used the same as wood products. Hempcrete is a recyclable building material where production is less energy-intensive and helps improve air quality (Small and Marcus 2002).

Additionally, hemp can be used in phytoremediation to clean contaminated soil in nuclear disaster sites, contaminated mines, and soils with heavy metals (Linger 2002). Industrial hemp bioaccumulates heavy metals, which could have useful applications worldwide. Phytoremediation using hemp can clean up many abandoned mines in Colorado, or areas with soil contamination (Citterio et al., 2003; Stonehouse et al., 2019). 
Cannabis sativa can also be grown to produce various phytochemicals. Most important are various cannabinoids, plant secondary metabolites that induce varied pharmacological effects in mammalian species including euphoric and psychotropic/medical effects (McCormick, 2017). When mammals consume or inhale cannabinoids after processing, the endocannabinoid systems in human brains activate and modulate several physiological processes (Gertsch et al., 2010). The cannabis plant has just over 100 isolated cannabinoids or plant secondary metabolites, but the best studied include tetrahydrocannabinol (THC), cannabidiol (CBD) and cannabinol (CBN) (ElSohly and Slade, 2005). Best known of the cannabinoids is tetrahydrocannabinol (THC) which has typically been used for recreational and medicinal purposes (Andre et al., 2016) and occurs in high concentrations in plants grown for marijuana. Cannabis sativa grown for production of hemp, as defined by U.S. law, contains low percentages of THC $(<0.3 \%$ by dry weight).

\section{Hemp CBD cultivars}

Hemp may be bred and developed to produce different plant materials, notably fiber, seed and non-euphoric phytochemicals. Chief among the latter is cannabidiol (CBD), and presently the great majority of hemp in Colorado is for production of this, or related, compounds. Cannabidiol is found primarily in the flowers and leaves of the hemp plant. It is one of many powerful cannabinoids that is may be psychoactive, and but does not produce euphoria or a feeling of being "high". Cultivars with CBD have been most recently developed to produce this compound for pharmaceutical use. CBD has a wide range of uses, and one formulation (Epidiolex ${ }^{\circledR}$ ) was approved by the FDA for treating severe forms of epilepsy (Hill 2015).

Cultivars grown for pharmaceutical purpose are typically all-female plants and are often cloned. CBD farmers use plant propagation to produce clonal hemp populations that sustain 
multiple pest populations. Non-fertilized, resinous flower buds are the primary harvested plant part. These varieties are being grown both in fields (in summer) and in indoor facilities (in winter). Most all hemp farmers in the state of Colorado are growing hemp for CBD as it is currently a high value crop and in-state processing facilities to handle fiber extraction are not available.

\section{Hemp seed/fiber cultivars}

Historically, hemp grown in the US was almost entirely produced for bast fiber, and this continues to represent a market for hempcrete, building materials, and textiles. In addition, multiuse hemp cultivars (e.g., oil seed, seed/fiber) are grown and sold into several markets (nutritional, biofuel, feed). Hemp seed oil contains healthy omega fatty acids (Callaway, 2004; Simopoulos, 2002). Cultivars grown to maximize oilseed yield are direct seeded and require pollination ( $C$. sativa is usually dioecious; some cultivars are monoecious). Cultivars grown for fiber are varieties that contain very low THC content $(=/<0.3 \%$ THC). Farmers seek stable seed so that they can produce hemp with consistent levels of cannabinoids (Galasso et al., 2016). Canada and Europe are now moving from an emphasis on fiber to focusing on oilseed. Currently there are massive efforts around the US to develop improved cultivars for production of one or more of all of these above commodities (Fike, 2016).

\section{Pesticide use in hemp}

The national legalization of industrial hemp through the 2018 Agricultural Improvement Act allows farmers nationwide to take advantage of growing hemp. To date, there are no insecticides that are federally approved or regulated for use on the hemp crop, which has created a troublesome situation. Pesticide use in hemp demands extreme reform regarding label language. The regulation of pesticides for cannabis crops such as marijuana and hemp have not 
been addressed at the federal level. Pesticide regulation occurs through the Environmental Protection Agency (EPA) and they will step in to regulate pesticide use in hemp when the 2020 the federal hemp program is launched in the near future.

Without federal hemp regulation, state departments of agriculture have been managing pesticide use by identifying which pesticides can be used legally in accordance with the Pesticide Applicators' Act and its rules in the production of cannabis (marijuana and industrial hemp). As a result, pesticide management now varies tremendously across state lines. The lack of federal enforcement has allowed for farmers and applicators to adhere less stringently to the label language of pesticides.

In the future, pesticides will likely be regulated differently between hemp for oil and seed compared with hemp grown for $\mathrm{CBD}$ extraction. One looming issue for $\mathrm{CBD}$ production is there is no consistent federal testing for insecticide residues in the extracted product. CBD will be much more heavily regulated considering it is grown for extractable compounds and extraction methods may concurrently extract certain pesticide residues (Raber et al., 2015). Today, the potential health risks regarding pesticides levels in extracted cannabis products is unknown unless materials are tested by private labs. Accurate and cost-effective analytical methods to determine pesticide residues in cannabis products is required as we move forward (Searfoss and Telepchak 2016). 


\section{REFERENCES}

Andre, C. M. et al. 2016. Cannabis sativa: The plant of the thousand and one molecules. Frontiers in Plant Science, 7, 19.

Anonymous. 1996. Car-maker turns to Cannabis — for fiber. Nature 384: 95

Anonymous. 1938. "New billion-dollar crop." Popular Mechanics Feb 238: 238

Booth, M. 2005. Cannabis: a History. Picador Reading Group Guides/St. Martins Press. New York. pp. 448.

Bowyer, J. L. 2001. Industrial hemp (Cannabis sativa L.) as a papermaking raw material in Minnesota: Technical, economic, and environmental considerations. Department of Wood and Paper Science, University of Minnesota. Report Series. 1-33.

https://pdfs.semanticscholar.org/0d3d/32a8cb3af4585b7c76de688b7caafb669f4c.pdf?.ga=2.929 $\underline{50613.9915998 .1572286852-1136249370.1570834466}$

Callaway, J. C. 2004. Hempseed as a nutritional resource: An overview. Euphytica, 140(1-2), 6572.

Citterio, S. et al. 2003. Heavy Metal Tolerance and Accumulation of Cd, Cr and Ni by Cannabis sativa L. Plant and Soil, 256(2), 243.

Cherney, J., and E. Small. 2016. Industrial hemp in North America: production, politics and potential. Agronomy. 6(4), 58.

Cranshaw, W. et al. 2019. Developing Insect Pest Management Systems for Hemp in the United States: A Work in Progress. Journal of Integrated Pest Management, 10(1), 26.

Dewey, L. H., and Merrill, J. L. 1916. Hemp hurds as paper-making material. Washington DC: US Department of Agriculture. No. 404

Deitch, R. 2003. Hemp: American history revisited: the plant with a divided history . Algora Publishing, New York. pp. 244.

ElSohly, M. A., and D.Slade. 2005. Chemical constituents of marijuana: the complex mixture of natural cannabinoids. Life Sciences, 78(5), 539-548. 
Fike, J. 2016. Industrial Hemp: Renewed Opportunities for an Ancient Crop. Critical Reviews in Plant Sciences 35: 406-424.

Galasso, I. et al. 2016. Variability in seed traits in a collection of Cannabis sativa L. genotypes. Frontiers in Plant Science, 7, 688.

Gertsch, J. et al. 2010. Phytocannabinoids beyond the Cannabis plant-do they exist? British Journal of Pharmacology, 160(3), 523-529.

Grotenhermen, F., and K. Müller-Vahl, 2016. Medicinal uses of marijuana and cannabinoids. Critical Reviews in Plant Sciences, 35(5-6), 378-405.

Hill, K. P. 2015. Medical marijuana for treatment of chronic pain and other medical and psychiatric problems: a clinical review. Journal of the American Medical Association, 313(24), 2474-2483.

Johnson, R. 2014. Hemp as an agricultural commodity. Library of Congress Washington DC Congressional Research Service. https://www.fas.org/sgp/crs/misc/RL32725.pdf pp. 44.

Li, H. L. 1973. An archaeological and historical account of cannabis in China. Economic Botany, 28(4), 437-448.

McCormick M. 2017. The health effects of cannabis and cannabinoids: The current state of evidence and recommendations for research. National Academies of Sciences, Engineering, and Medicine. Committee of the Health Effects of Marijuana: An Evidence Review and Research Agenda. National Academies Press. 1-4 http://nationalacademies.org/hmd/reports/2017/healtheffects-of-cannabis-and-cannabinoids.aspx

Raber, J.C., S. Elzinga, and C. Kaplan. 2015. Understanding dabs: contamination concerns of cannabis concentrates and cannabinoid transfer during the act of dabbing. J. Toxicol. Sci. 40:797-803.

Pain, S. 2015. A potted history. Nature, 525(7570), S10.

Rull, V., and T. Vegas-Vilarrúbia. 2014. Preliminary report on a mid-19th century Cannabis pollen peak in NE Spain: Historical context and potential chronological significance. The Holocene, 24(10), 1378-1383.

Salentijn, E. M. et al. 2015. New developments in fiber hemp (Cannabis sativa L.) breeding. Industrial crops and products, 68, 32-41. 
Simopoulos, A. P. 2002. The importance of the ratio of omega-6/omega- 3 essential fatty acids. Biomedicine and Pharmacotherapy. 56(8), 365-379.

Small, E., and D. Marcus. 2002. Hemp: A New Crop with New Uses for North America. In Trends in New Crops and New Uses. J. Janick and A. Whipkey (eds.). American Society for Horticultural Science, Alexandria, VA. pp 284-326

Small, E. 2015. Evolution and classification of Cannabis sativa (marijuana, hemp) in relation to human utilization. The botanical review. 81(3), 189-294.

Stonehouse, G. C. 2019. Phytoremediation and biofortification potential of Cannabis sativa $\mathrm{L}$. M.S. Thesis, Colorado State University Libraries.

54pp.https://mountainscholar.org/bitstream/handle/10217/195371/Stonehouse_colostate_0053N 15414.pdf?sequence=1

Willsie, C.P. et al. 1942. Hemp - A War Crop for Iowa. Bulletin 49 Iowa Agricultural Experiment Station. 13 pp. http://www.globalhemp.com/1942/01/hemp-a-war-crop-foriowa.html

Wang X., D. Mackowsky, J. Searfoss, and M. J. Telepchak. 2016. Determination of Cannabinoid Content and Pesticide Residues in Cannabis Edibles and Beverages. Cannabis Science and Technology Magazine, Special Issues. Volume 34, Issue 10, pg 20-27 http://www.cannabissciencetech.com/article/determination-cannabinoid-content-and-pesticideresidues-cannabis-edibles-and-beverages 


\section{CHAPTER 2 - A SURVEY OF THE ARTHROPOD FAUNA ASSOCIATED WITH HEMP (CANNABIS SATIVA) GROWN IN EASTERN COLORADO}

With the recent reemergence of Cannabis sativa-derived crops in the United States, there is tremendous demand for credible information regarding all aspects of its production, including

those related to pest management. One prior report of arthropods on hemp found approximately 300 species associated with the crop (McPartland, 1996). Historically, within the United States very few insect studies have been conducted with involving hemp insects due to lack of a commercial industry, resulting from federal restrictions (Lago and Stanford, 1989). A worldwide review of hemp insects (McPartland et al., 2000) provides an excellent resource on the status of information on insects associated with the crop, although few of the references relate to North American species. Many phytophagous species were observed infesting hemp in 2016, 2017, and 2018 on various hemp cultivars across Colorado. The arthropod pests found associated with hemp grown in eastern Colorado during this study period were described, along with their general biology and association with the crop, with particular emphasis on identifying those species that most directly affect the production of the crop.

\section{Methods and materials}

Commercial plantings of hemp (Cannabis sativa L.) were surveyed in Colorado for insects during the 2016, 2017, and 2018 growing seasons. A total of 21 fields, present in eight eastern Colorado counties, were visited during this period (Table 2.1), five of which (Sites $1,3,6,7,11)$ were sampled all three years of the sampling period. Most fields surveyed involved Colorado hemp producers, registered by the Colorado Department of Agriculture. In addition, fields at two sites (Sites 1, 3), were variety trials maintained at Colorado State University Agricultural Experiment Stations and managed by the Colorado Department of Agriculture. 
Table 2.1 Location of field sites in eastern Colorado and the type of hemp crop grown involved in hemp field surveys, Spring 2016 - Fall 2018. Cultivars produced for seed/fiber/grain are indicated with the color orange and cultivars produced for cannabidiol (CBD) are indicated with the color blue. Coordinates were provided through Google Earth Pro version 7.3.2.5776

\begin{tabular}{|c|c|c|c|c|}
\hline Field Site \# & Type of hemp & County & Latitude & Longitude \\
\hline Site 1 & Seed/Fiber/Grain & Larimer & $40^{\circ} 36^{\prime} 39.55^{\prime \prime} \mathrm{N}$ & $104^{\circ} 59^{\prime} 48.11^{\prime \prime W}$ \\
\hline Site 2 & Seed/Fiber/Grain & Larimer & $40^{\circ} 39^{\prime} 16.45^{\prime \prime} \mathrm{N}$ & $104^{\circ} 59^{\prime} 55.91^{\prime W}$ \\
\hline Site 3 & Seed/Fiber/Grain & Otero & $38^{\circ} 2^{\prime} 22.76 " \mathrm{~N}$ & $103^{\circ} 41^{\prime} 33.50^{\prime \prime W}$ \\
\hline Site 4 & Seed/Fiber/Grain & Washington & $40^{\circ} 7 ' 23.64 " \mathrm{~N}$ & $102^{\circ} 53^{\prime} 5.35^{\prime \prime} \mathrm{W}$ \\
\hline Site 5 & Seed/Fiber/Grain & Weld & $40^{\circ} 27^{\prime} 55.82^{\prime \prime N}$ & $104^{\circ} 32^{\prime} 53.40^{\prime \prime W}$ \\
\hline Site 6 & CBD & Weld & $40^{\circ} 31^{\prime} 49.18^{\prime \prime} \mathrm{N}$ & $104^{\circ} 34^{\prime} 0.06^{\prime \prime W}$ \\
\hline Site 7 & CBD & Pueblo & $38^{\circ} 15^{\prime} 37.30^{\prime \prime} \mathrm{N}$ & $104^{\circ} 27^{\prime} 39.46^{\prime \prime W}$ \\
\hline Site 8 & CBD & Larimer & $40^{\circ} 35^{\prime} 28.81^{\prime \prime} \mathrm{N}$ & $105^{\circ} 7^{\prime} 46.02^{\prime \prime W}$ \\
\hline Site 9 & CBD & Weld & $40^{\circ} 19^{\prime} 52.07 " \mathrm{~N}$ & $104^{\circ} 53^{\prime} 5.39^{\prime \prime} \mathrm{W}$ \\
\hline Site 10 & CBD & Otero & $38^{\circ} 00^{\prime} 24.92^{\prime \prime} \mathrm{N}$ & $103^{\circ} 31^{\prime} 19.75^{\prime \prime W}$ \\
\hline Site 11 & CBD & Otero & $38^{\circ} 6{ }^{\prime} 3.05^{\prime \prime} \mathrm{N}$ & $103^{\circ} 47^{\prime} 57.17^{\prime \prime W}$ \\
\hline Site 12 & CBD & Otero & $38^{\circ} 3{ }^{\prime} 31.67 " \mathrm{~N}$ & $103^{\circ} 45^{\prime} 14.00^{\prime \prime W}$ \\
\hline Site 13 & $\mathrm{CBD}$ & Yuma & $40^{\circ} 1^{\prime} 41.87^{\prime \prime} \mathrm{N}$ & $102^{\circ} 14^{\prime} 31.19^{\prime \prime W}$ \\
\hline Site 14 & $\mathrm{CBD}$ & Weld & $40^{\circ} 16^{\prime} 42.50 " \mathrm{~N}$ & $104^{\circ} 42^{\prime} 36.26^{\prime \prime W}$ \\
\hline Site 15 & CBD & Weld & $40^{\circ} 21^{\prime} 25.19^{\prime \prime} \mathrm{N}$ & $104^{\circ} 42^{\prime} 3.97^{\prime \prime W}$ \\
\hline Site 16 & CBD & Adams & $39^{\circ} 42^{\prime} 0.36^{\prime \prime} \mathrm{N}$ & $104^{\circ} 19^{\prime} 14.79^{\prime \prime W}$ \\
\hline Site 17 & CBD & Yuma & $40^{\circ} 5^{\prime} 10.82^{\prime \prime} \mathrm{N}$ & $102^{\circ} 20^{\prime} 46.93 " \mathrm{~W}$ \\
\hline Site 18 & $\mathrm{CBD}$ & Boulder & $40^{\circ} 10^{\prime} 20.24^{\prime \prime} \mathrm{N}$ & $105^{\circ} 11^{\prime} 45.28^{\prime \prime W}$ \\
\hline Site 19 & CBD & Weld & $40^{\circ} 19^{\prime} 52.07 " \mathrm{~N}$ & $104^{\circ} 53^{\prime} 5.39^{\prime \prime} \mathrm{W}$ \\
\hline Site 20 & CBD & Weld & $40^{\circ} 23^{\prime} 25.98^{\prime \prime N}$ & $104^{\circ} 35^{\prime} 20.70^{\prime \prime W}$ \\
\hline - Site 21 & CBD & Otero & $38^{\circ} 00^{\prime} 27.68^{\prime \prime} \mathrm{N}$ & $103^{\circ} 37^{\prime} 3.93^{\prime \prime W}$ \\
\hline
\end{tabular}




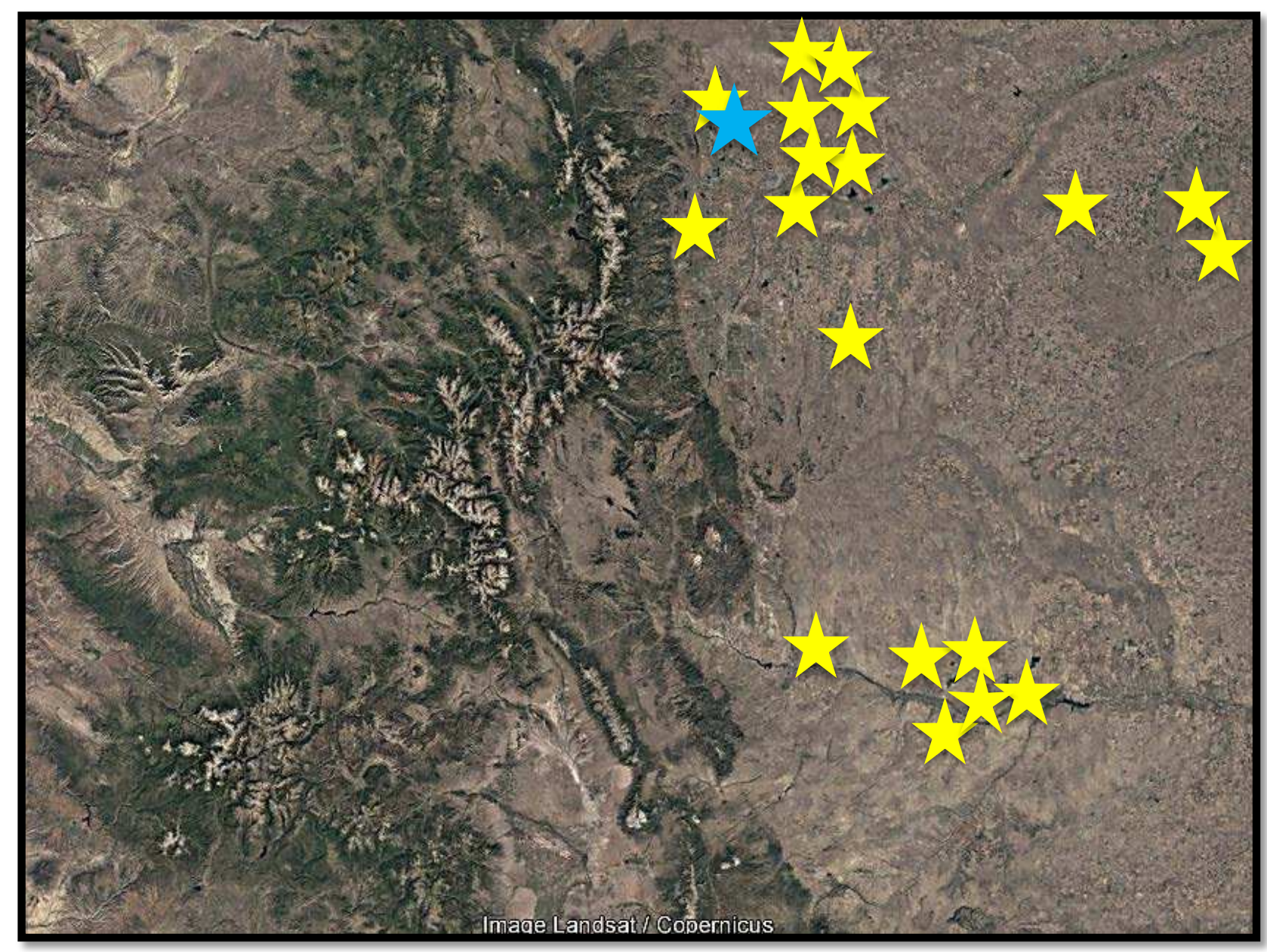

Figure 2.1 Map of Colorado with twenty-one sample locations indicated by the yellow stars sampled in 2016, 2017 and 2018. The blue star indicates the location of Fort Collins Colorado, the location of Colorado State University. Map generated through Google Earth Pro version 7.3.2.5776 using Landsat satellite imagery.

\section{Farming Methods}

An extremely wide range of methods was observed to be used by growers to produce hemp crops in eastern Colorado during the study period. Planting dates ranged from late-May to mid-June across all site locations. Harvesting occurred around early October each year for all sites. All fields were irrigation, by irrigation methods utilized varied and included drip systems, center pivots with sprinklers, to furrow irrigation. Cultivar type, planting density, row spacing, soil amendments, and soil fertility were not standardized variables across sites due to differing land managers. 
Essentially all hemp grown primarily for production of CBD were derived from clones propagated from cuttings of mother plants and grown as transplants in greenhouses before transplanting outdoors to fields. Some CBD sites utilized portable and permanent growing containers made from custom, non-woven polypropylene material which is highly durable. Cultivars grown for seed or fiber were direct seeded into cultivated fields.

Agricultural weeds were abundant at many sites (Table 2.2), a situation that is more common in hemp than many other crops because there is very limited availability of herbicides registered for use on the crop. Weeds that were most commonly observed included field bindweed (Convolvus arvensis), redroot pigweed (Amaranthus retroflexus) and common lambsquarters (Chenopodium berlandieri).

Table 2.2 Agricultural weeds found commonly in Colorado hemp fields surveyed in 2016, 2017, 2018.

\begin{tabular}{llll} 
Family & Scientific Name & Common name & Type \\
Amaranthaceae & Amaranthus retroflexus & Redroot pigweed & Introduced annual \\
Asteraceae & Ambrosia tomentosa & Skeleton bursage & Native perennial \\
Asteraceae & Cirsium arvense & Canada thistle & Introduced perennial \\
Asteraceae & Helianthus annuus & Common sunflower & Native annual \\
Asteraceae & Taraxacum officinale & Dandelion & Introduced annual \\
Asteraceae & Sonchus arvensis & Sowthistle & Introduced perennial \\
Chenopodiaceae & Bassia scoparia & Kotchia & Introduced annual \\
Chenopodiaceae & Chenopodium berlandieri & Lambsquarters & Introduced annual \\
Chenopodiaceae & Chenopodium glaucum & Oakleaf goosefoot & Introduced annual \\
Convolvulaceae & Convolvus arvensis & Field bindweed & Introduced perennial \\
Euphorbiaceae & Chamaesyce maculata & Spotted spurge & Native annual \\
Fabaceae & Medicago lupulina & Black medic & Introduced annual \\
Malvaceae & Malva neglecta & Common mallow & Introduced annual \\
Poaceae & Digitaria sanguinalis & Large crabgrass & Introduced annual \\
Poaceae & Echinochloa crus galli & Barnyardgrass & Introduced annual \\
Portulacaeae & Portulacha oleracea & Common purslane & Introduced annual \\
Solanaceae & Quincula lobata & Chinese lantern & Native perennial \\
Solanaceae & Solanum physalifolium & Hairy nightshade & Introduced annual \\
Solanaceae & Solanum rostratum & Buffalobur & Introduced annual \\
Solanaceae & Solanum triflorum & Cutleaf nightshade & Native annual \\
Zygophyllaceae & Tribulus terrestris & Puncturevine & Introduced annual \\
& & & \\
\hline
\end{tabular}




\section{Sampling Methods}

Survey plots represented a broad geographic area of the High Plains of eastern Colorado. During each visit at a hemp field, the standard sampling method used was to take 100-500 sweep net samples from the hemp canopy with a 15 inch-diameter sweep net (Figure 2.3). An effort was made to sample from the time range of approximately 9:00 a.m. to 11:00 a.m. MDT when the weather was moderately warm. By sampling in the morning, insects were relatively still while resting within the hemp canopy enabling the sweep netter to collect more flying insects. Insect samples were taken by walking hemp rows while vigorously sweeping the net across the hemp plants. Only two assigned sweep netters were used for all samples taken over the three-year study to standardize this variable as much as possible.

A weakness with using a sweep net includes plant size and time of year. In the early months plants are small, so the full plant was sampled. The issue arose when hemp plants were older and larger so only a portion of that plant could be swept from ground level. In result, as the season progressed we were able to only sample a part of the whole plant as they were over 12 feet tall. To accomplish this, larger plants were shaken vigorously over the sweep net using the beat-sheeting method.

Fields were sampled for arthropods weekly, biweekly, or monthly for counties within a 50-mile radius and whenever possible for counties over a 100 mile radius from Fort Collins. The most intensive sampling was conducted during the third year of this study (2018). In 2016 there were a total of 17 samples taken, 52 samples in 2017, and 80 samples in 2018.

Insects were then placed into a plastic Ziploc ${ }^{\circledR}$ bag and brought back to the lab for identification, labeling, and pinning. The majority of specimens were initially stored in collection vials filled entirely with $95 \%$ ethanol until insects were identified and quantified. 


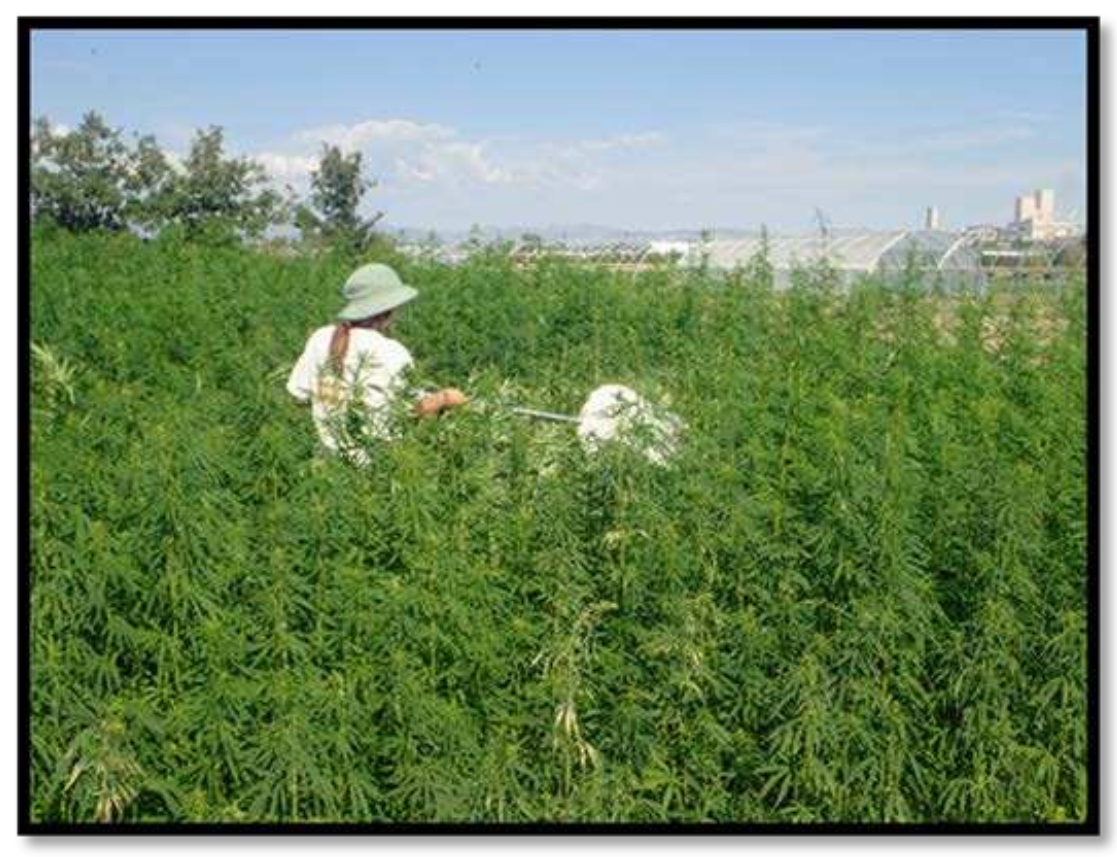

Figure 2.2 Use of a sweep net to sample industrial hemp for arthropods at the Colorado State University Agricultural Research, Development, and Education Center (ARDEC) facility in Larimer County, 7 July, 2018.

Various other sampling methods were used in addition to sweep sampling. Individual arthropods observed during field surveys were often hand collected for further rearing and/or identification. This method was also used to obtain photographs of specimens, as photographic records of hemp insects were needed for producing educational materials for the outreach aspects associated with these studies. Observational notes were recorded at each site visit when relevant. After initial observation and photography occurred, both sides of each leaf on the chosen hemp plant were scanned; as were the petioles, axils, and stems (Pedigo and Buntin, 1993). The apical leaves were searched for mites, plant bugs, thrips, small arthropods, and arachnids. Flowers and buds were also searched when present for damage, pathogens, pollen feeders or insect retreats.

A Berlese funnel was utilized in the last year of study. Berlese funnels were used in 2018 for extracting arthropods from soil and litter samples from hemp plots that would otherwise go 
unnoticed by an observer. These samples were important in isolating small arthropods that would be missed by a sweep net. The sample was placed within a funnel on a mesh screen, exposed to a light source immediately above, and a collection container containing ethanol was placed at the bottom of the funnel. The light source forces arthropods from the drying sample to move downward within the funnel where they ultimately fall into the collecting container of ethanol.

To categorize all arthropods found in hemp, individuals were placed into their related feeding guilds to indicate their association to the hemp crop. Most species found were herbivorous on hemp. Herbivores that were associated with hemp were categorized as being defoliators, stem borers, chewers of buds/flowers and/or developing seed, root feeders, leafminers, fluid feeder of foliage, fluid feeder of buds, flowers, and/or developing seeds, and/or root fluid feeders. Herbivores associated with hemp pollen comprised another group associated with the crop, including bees and other pollen feeding insect groups. Natural enemies of arthropods were a separately distinguished group. Other arthropods present in fields included scavengers/detritivores collected on hemp; incidental species on hemp (herbivore on other crops); incidental species on hemp (herbivore on weeds or unknown hosts).

All species collected were identified to the family level via taxonomic keys, particularly those of Triplehorn and Johnson (2005) and Stehr (1987). In addition, many specimens were taken to genus or species wherever possible. Many specimens were identified using the resources of the C.P. Gillette Museum of Arthropod Diversity, often with the assistance of its director, Dr. Boris Kondratieff. Efforts were also made to determine the association of the collected arthropods with hemp. 


\section{Statistical Methods}

There were two statistical analysis preformed for this observational study. The first involved simple summary statistics of the collected arthropods to calculate proportions of different kinds of hemp arthropods collected in sweep net samples in 2016, 2017, and 2018. Proportions are also reported by order and by their associated feeding guilds.

A separate set of analyses were performed to compare the relative diversity of the arthropods present in different types of hemp. These involved comparisons of collections made in CBD hemp sites to those in sites being grown for seed or fiber over the three-year study using the Shannon-Wiener Diversity Index. The Shannon-Wiener index diversity measure $\left(H^{\prime}=-\sum\right.$ pi lnpi $R i=1)$ generates values $(\mathrm{H})$ representing site diversity which were compared directly to one another. This index combines evenness and richness into a single measure where $\mathrm{p}$ is the proportion $(\mathrm{n} / \mathrm{N})$ of individuals belonging to one species (n) found in the collection. $\mathrm{N}$ is the total number of individuals (N) found in the sample (Southwood and Henderson 2000). Once diversity indices were calculated for each sample date, the samples were averaged across the month of collection for each site location. Site to site differences were first analyzed using an ANOVA model created using the 'emmeans' and 'dplyr' packages in R version 3.5.3 (R Core Team 2019; Ott and Longnecker, 2010). Pairwise comparisons of means for site diversity are displayed using a cld displaying groups 


\section{Results and discussion}

Fifteen arthropod orders were determined from hemp in the three-year sampling period in Colorado. A total of 73 arthropod families were found representing 142 genera (Table 2.4). Arthropod samples were collected from 21 different locations over the study, with site locations varying year to year. After collecting insects in 2017 and 2018, an abundance of natural enemies, scavengers, and incidental species were documented, and proportions are reported (Table 2.6).

Hemiptera (40\%) were the most dominant order of insects collected in samples of eastern Colorado hemp fields. Coleoptera ( 20\%), Diptera ( 20\%), Thysanoptera (7\%), Hymenoptera $(10 \%)$ and Araneae (4\%) were also collected abundantly at all sites. Seven of the sixteen identified orders were spatially rare (lower than 1\%): Collembola (0.02\%), Lepidoptera (0.3\%), Mantodea (0.01\%), Odonata (0.02\%), Opiliones (0.5\%), and Dermaptera, $(0.2 \%)$ (Table 2.4; Table 2.5).

Field samples extracted with a Berlese funnel were made in 2018 on a limited number of samples from Larimer and Weld counties (Table 2.3). In these samples, eighteen families were found representing eight orders. The western flower thrips, Frankliniella occidentalis, (Thysanoptera: Thripidae) made up almost $40 \%$ of the total Berlese samples in 2018. The cannabis aphid, Phorodon cannabis, was easily sampled using Berlese funnel extraction and they made up $30 \%$ of the total sample. Berlese funnel extractions did identify several insects that were not found in sweep net samples. 
Table 2.3 Arthropods collected from the Berlese funnel (2018 only)

\begin{tabular}{lllllll} 
& & & & 2018 & Percent \\
Order & Family & Genus & species & Life Stage & $\mathbf{1 1 7 2}$ & $\mathbf{1 0 0 \%}$ \\
Acari & Tetranychidae & Tetranychus & urticae & Adult & 1 & 0.08 \\
& - & - & - & Adult & 25 & 2.12 \\
Coleoptera & Phalacridae & Olibrus & sp. & Adult & 6 & 0.5 \\
Collembola & Symphypleona & - & - & Adult & 3 & 0.25 \\
Dermaptera & Forficulidae & Forficula & auricularia & Adult & 4 & 0.34 \\
Diptera & Chironomidae & - & - & Adult & 1 & 0.08 \\
& Cecidomyiidae & & Larvae & 32 & 2.73 \\
Hemiptera & Anthocoridae & Orius & insidiosus & Adult/Nymph & 174 & 14.9 \\
& Aphidae & Phorodon & cannabis & Adult/Nymph & 354 & 30.2 \\
& Cicadellidae & Ceratagallia & uhleri & Adult/Nymph & 7 & 0.6 \\
& Lygaeidae & Nysiusra & phanus & Adult/Nymph & 25 & 2.12 \\
& Nabidae & Nabis & alternatus & Adult & 8 & 0.7 \\
& Miridae & Lygus & elisus & Adult/Nymph & 22 & 1.9 \\
& Miridae & Chalamydatus & associatus & Adult/Nymph & 45 & 3.83 \\
Hymenoptera & Aphidiinae & Aphidius & ervi & Adult & 3 & 0.25 \\
& Chalcididae & - & Adult & 5 & 0.42 \\
& Formicidae & Solenopsis & sp. & Adult & 10 & 0.85 \\
Orthoptera & Gryllidae & Oecanthus & sp. & Nymph & 1 & 0.08 \\
Thysanoptera & Thripidae & Frankliniella & occidentalis & Adult & 446 & 38.1 \\
& & & & & \\
\hline
\end{tabular}

${ }^{1}$ Species undetermined. Larvae were predaceous species thought to be associated with mites (Feltiella acarisuga) and/or aphids (Aphidoletes aphidimyza). 
Table 2.4 Arthropods collected from hemp (Cannabis sativa) fields in eastern Colorado, Spring 2016-Fall 2018. All quantified samples were collected by sweep net. Species not quantified and marked "_" or "N/A" were observed on the crop directly during field surveys or through subsequent Berlese funnel extraction.

Total number

Association

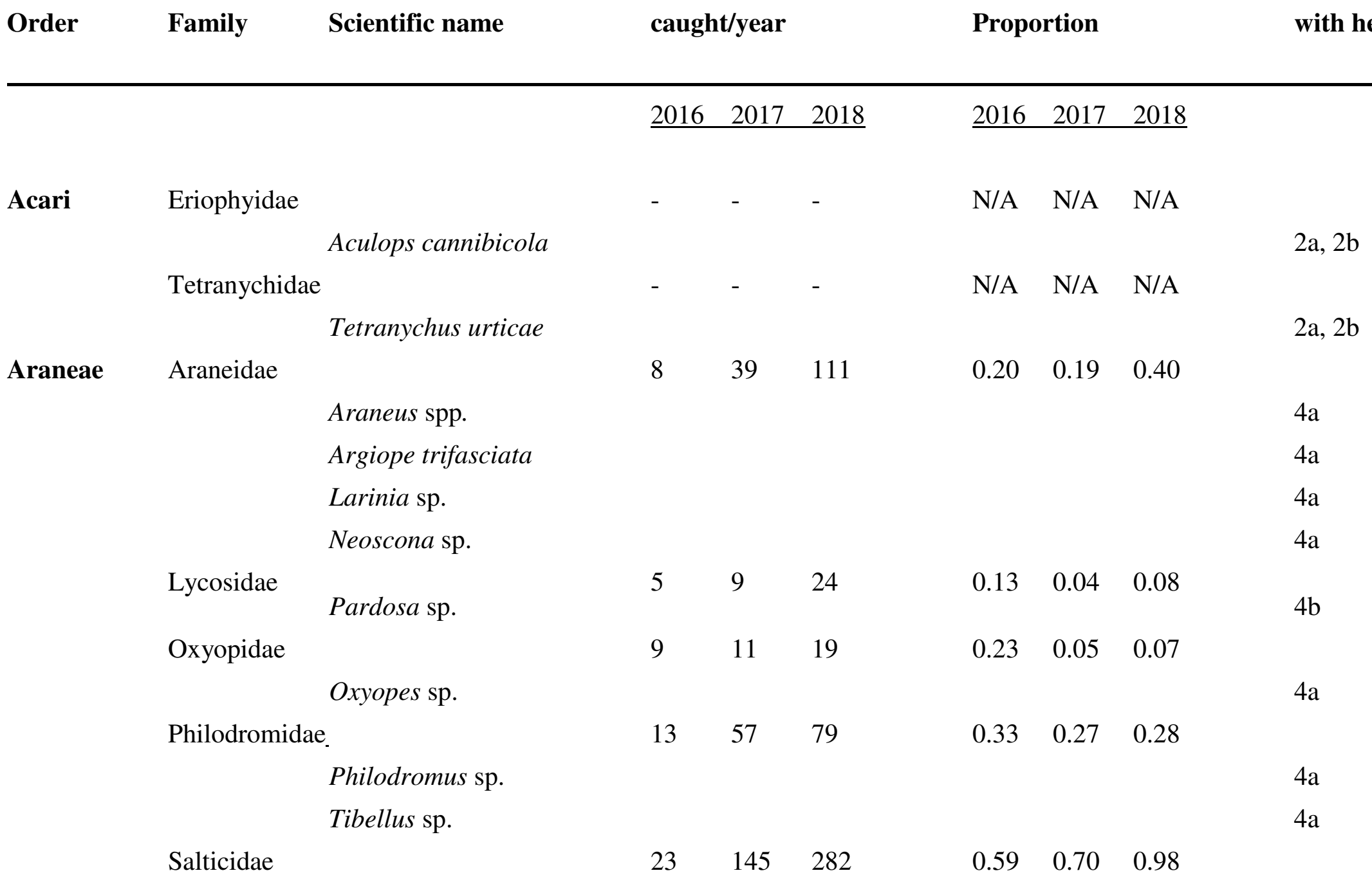


Eris sp.

Pelegrina sp.

Phidippus audax

$4 \mathrm{a}$

Phidippus clarus

Phidippus spp.

$4 \mathrm{a}$

Sassacus papenhoei

$4 a$

Sitticus sp.

Tetragnathidae

Tetragnatha sp.

Thomisidae

Mecaphesa celer

\section{Coleoptera}

Cantharidae

Chrysomelidae $\begin{array}{lll}11 & 34 & 38\end{array}$

59

$316 \quad 407$

0

$25 \quad 21$

272

1698

2152

$\begin{array}{lll}6.94 & 8.07 & 7.50\end{array}$

Altica sp.

Charidotella sexpunctata

Chelymorpha cassidea

Deloyala guttata

Diabrotica undecimpunctata

Diabrotica virgifera

1a, 6a, 6b

Disonycha triangularis

$6 a$

Leptinotarsa decemlineata

$6 \mathrm{~b}$

Phyllotreta pusilla

$6 b$

Systena blanda 


\begin{tabular}{|c|c|c|c|c|c|c|c|c|}
\hline \multirow[t]{9}{*}{ Coccinellidae } & & 89 & 866 & 1168 & 2.26 & 4.12 & 4.07 & \\
\hline & Chilocorus sp. & & & & & & & $4 \mathrm{~b}$ \\
\hline & Coccinella septempunctata & & & & & & & $4 a$ \\
\hline & Coccinella novemnotata & & & & & & & $4 a$ \\
\hline & Coccinella transversogutta & & & & & & & $4 \mathrm{~b}$ \\
\hline & Harmonia axyridis & & & & & & & $4 b$ \\
\hline & Hippodamia convergens & & & & & & & $4 \mathrm{~b}$ \\
\hline & Hippodamia parenthesis & & & & & & & $4 \mathrm{~b}$ \\
\hline & Olla v-nigrum & & & & & & & $4 \mathrm{~b}$ \\
\hline \multirow[t]{3}{*}{ Lampyridae } & & 8 & 28 & 11 & 0.20 & 0.13 & 0.04 & \\
\hline & Pyropyga minuta & & & & & & & \\
\hline & Pyropyga spp. & & & & & & & \\
\hline \multirow[t]{3}{*}{ Melyridae } & & 98 & 425 & 915 & 2.50 & 2.02 & 3.20 & \\
\hline & Collops bipunctatus & & & & & & & $4 a$ \\
\hline & Malachius aeneus & & & & & & & $3 b$ \\
\hline \multirow[t]{3}{*}{ Mordellidae } & & 26 & 152 & 202 & 0.66 & 0.72 & 0.70 & \\
\hline & Mordella sp. & & & & & & & $3 b$ \\
\hline & Mordellistena sp. & & & & & & & $3 b$ \\
\hline \multirow[t]{2}{*}{ Anthicidae } & & 4 & 44 & 38 & 0.10 & 0.21 & 0.13 & \\
\hline & Notoxus sp. & & & & & & & $3 b, 4 b$ \\
\hline \multirow[t]{2}{*}{ Phalacridae } & & 21 & 114 & 78 & 0.53 & 0.54 & 0.27 & \\
\hline & Olibrus sp. & & & & & & & $5 \mathrm{a}$ \\
\hline \multirow[t]{2}{*}{ Scarabaeidae } & & 1 & 0 & 3 & 0.02 & 0 & 0.01 & \\
\hline & Euphoria inda & & & & & & & $5 \mathrm{a}$ \\
\hline
\end{tabular}


Collembola Symphypleona

Dermaptera Forficulidae

Forficula auricularia

Diptera

Agromyzidae

Agromyza sp.

Liriomyza sp.

Asilidae

Diogmites sp.

Efferia sp.

Proctocanthus sp.

Bombylidae

Bombylius sp.

Systoechus sp.

Villa sp.

Cecidomyiidae

Aphidoletes aphidimyza

Feltiella acarisuga

Chironomidae

Chloropidae

Apallates sp.

Chlorops sp.

Meromyza sp.

Thaumatomyia glabra

$\begin{array}{lll}0 & 0 & 5 \\ 0 & 0 & 4\end{array}$

$\begin{array}{lll}0 & 0 & 4\end{array}$

$255 \quad 528 \quad 1092$

$\begin{array}{lll}6.50 & 2.51 \quad 3.81\end{array}$

10

$19 \quad 34$

34

$\begin{array}{lll}0 & 0 & 0.02\end{array}$

$5 a$

$1 \mathrm{a}, 4 \mathrm{a}$

$10-19-34$

$\begin{array}{lll}0.25 & 0.09 & 0.12\end{array}$

le

$1 \mathrm{e}$

$4 \mathrm{~b}$

$4 b$

$4 \mathrm{~b}$

$1 \quad 6 \quad 11$

$\begin{array}{lll}0.02 & 0.03 & 0.04\end{array}$

$4 b$

$4 b$

$4 \mathrm{~b}$

- $\quad$ - $\quad$ N/A N/A N/A

\begin{tabular}{|c|c|c|c|c|c|}
\hline 304 & 619 & 688 & 7.75 & 3.00 & 2.40 \\
\hline 266 & 1079 & 1565 & 6.78 & 5.13 & 5.45 \\
\hline
\end{tabular}

Dolichopodidae
63 $\begin{array}{lll}1.16 & 1.16 & 0.68\end{array}$ 
Condylostylus sp.

Hybotidae

$196 \quad 628 \quad 469$

$\begin{array}{lll}5.00 & 2.98 & 1.63\end{array}$

Platypalpus sp.

Lauxanidae

$\begin{array}{lll}- & - & 429\end{array}$

N/A N/A 1.49

Camptoprosopella sp.

Pipunculidae

Syrphidae

$\begin{array}{lll}3 & 8 & 16\end{array}$

$\begin{array}{lll}0.08 & 0.04 & 0.06\end{array}$

$4 a$

Eristalis tenax

$\begin{array}{lll}6 & 272 & 512\end{array}$

$\begin{array}{lll}0.15 & 1.29 & 1.78\end{array}$

Melanostoma mellinum

Toxomerus sp.

\section{Tipulidae}

Ulidiidae

$\begin{array}{lll}0 & 3 & 1 \\ 17 & 23 & 53\end{array}$

$\begin{array}{lll}0 & 0.01 & 0.003\end{array}$

$\begin{array}{lll}0.43 & 0.11 & 0.18\end{array}$

Physiphora demandata

$5 a$

$5 a$

$5 a$

$4 a$

$4 a$

$5 a$

$5 a$

\section{Hemiptera}

$\begin{array}{ll}\text { Anthocoridae } & \\ & \text { Orius insidiosus } \\ \text { Aphididae } & \\ & \text { Phorodon cannabis } \\ & \text { Aphis fabae } \\ & \text { Rhopaliiphum abdominalis }\end{array}$

$$
309 \quad 1409 \quad 1571
$$$$
\begin{array}{lll}
7.88 & 6.70 & 5.47
\end{array}
$$$$
241 \quad 1693 \quad 2320
$$$$
\begin{array}{lll}
6.14 & 8.45 \quad 8.37
\end{array}
$$

Berytidae

$\begin{array}{lll}3 & 10 & 7\end{array}$

$\begin{array}{lll}0.08 & 0.04 & 0.02\end{array}$

$$
\text { Jalysus wickhami }
$$

Cicadellidae

$202 \quad 1056 \quad 1602$
$2 \mathrm{a}$

$2 \mathrm{a}$

$2 c$

$4 a$ 
Ceratagallia uhleri

Agallia sp.

$2 \mathrm{a}$

Amblysellus grex

$2 \mathrm{a}$

Balclutha neglecta

$2 \mathrm{a}$

Cuerna sp.

$2 \mathrm{a}$

Empoasca sp.

$2 \mathrm{a}$

Endria inimical

$2 \mathrm{a}$

Graphocephala sp.

$2 \mathrm{a}$

Idiocerus sp.

$2 \mathrm{a}$

Macrosteles sp.

$2 \mathrm{a}$

Opsius stactogalus

$2 \mathrm{a}$

Paraphlepsius irroratus

$2 \mathrm{a}$

Texananus sp.

$2 \mathrm{a}$

Xerophloea sp.

Cixiididae

\section{9}

186

Melanoliarus sp.

Geocoridae

Geocoris punctipes

Lygaeidae

Lygaeus kalmii

Nysius raphanus

Miridae

Lygus spp.

Lygus elisus

Lygus hesperus

Lygus lineolaris

$26 \quad 86 \quad 370$

$230 \quad 1072 \quad 1563$ $\begin{array}{lll}0.23 & 0.13 \quad 0.64\end{array}$

$2 \mathrm{a}$

$2 a$

$4 a$

$6 b$

$2 \mathrm{a}, 2 \mathrm{~b}, 6 \mathrm{~b}$

$2 \mathrm{a}, 2 \mathrm{~b}$

$2 \mathrm{a}, 2 \mathrm{~b}$

$2 \mathrm{a}, 2 \mathrm{~b}$ 
Miridae other

\begin{tabular}{|c|c|c|c|c|c|c|c|c|}
\hline & Chalamydatus associatus & 255 & 1167 & 1215 & 6.50 & 5.55 & 4.38 & $4 a$ \\
\hline \multirow[t]{3}{*}{ Membracidae } & & 13 & 57 & 119 & 0.33 & 0.27 & 0.41 & \\
\hline & Micrutalis calva & & & & & & & $2 \mathrm{a}$ \\
\hline & Stictolobus sp. & & & & & & & $2 \mathrm{a}$ \\
\hline \multirow[t]{2}{*}{ Nabidae } & & 177 & 1503 & 1643 & 4.51 & 7.15 & 5.72 & \\
\hline & Nabis alternatus & & & & & & & $4 \mathrm{a}$ \\
\hline \multirow[t]{6}{*}{ Pentatomidae } & & 13 & 61 & 93 & 0.33 & 0.29 & 0.32 & \\
\hline & Chlorochroa ligata & & & & & & & $2 \mathrm{a}, 2 \mathrm{~b}$ \\
\hline & Chlorochroa sayi & & & & & & & $2 \mathrm{a}, 2 \mathrm{~b}$ \\
\hline & Euschistus sp. & & & & & & & $2 \mathrm{a}, 2 \mathrm{~b}$ \\
\hline & Perillus bioculatus & & & & & & & $4 a$ \\
\hline & Thyanta custator & & & & & & & $2 \mathrm{a}, 2 \mathrm{~b}$ \\
\hline \multirow[t]{4}{*}{ Reduviidae } & & 10 & 31 & 33 & 0.04 & 0.13 & 0.12 & \\
\hline & Phymata americana & & & & & & & $4 a$ \\
\hline & Sinea diadema & & & & & & & $4 \mathrm{a}$ \\
\hline & Zelus tetracanthus & & & & & & & $4 \mathrm{a}$ \\
\hline \multirow[t]{3}{*}{ Rhopalidae } & & 0 & 71 & 48 & 0 & 0.33 & 0.16 & \\
\hline & Liorhyssus hyalinus & & & & & & & $2 b$ \\
\hline & Harmostes reflexulus & & & & & & & $6 b$ \\
\hline \multirow[t]{3}{*}{ Tingidae } & & 0 & 0 & 8 & 0 & 0 & 0.03 & \\
\hline & Corythucha sp. & & & & & & & $6 b$ \\
\hline & Gargaphia sp. & & & & & & & $2 a, 6 b$ \\
\hline
\end{tabular}




\section{Hymenoptera}

Apidae

10

$511 \quad 692$

$\begin{array}{lll}2.67 & 2.43 & 2.41\end{array}$

Andrena helianthi

$3 a$

Anthophora montana

$3 \mathrm{a}$

Apis mellifera

$3 \mathrm{a}$

Bombus griseocollis

$3 \mathrm{a}$

Bombus fervidus

$3 a$

Bombus huntii

$3 \mathrm{a}$

Bombus pensylvanicus

$3 a$

Melissodes agilis

$3 a$

Melissodes bimaculatus

$3 a$

Melissodes sp.

$3 a$

Peponapis pruinosa

$3 a$

Svastra obliqua

Argidae

$21 \quad 66 \quad 83$

$\begin{array}{lll}0.53 & 0.31 & 0.28\end{array}$

Schizocerella pilicornis

Chalcidoidea

Chrysididae

Formicidae

Solenopsis sp.

Halictidae

Agapostemon angelicus 27

$\begin{array}{lll}749 & 1082 \\ 2 & 5 & 6 \\ 0 & 4 & 0\end{array}$

N/A $3.56 \quad 3.80$

$6 \mathrm{~b}$

$4 \mathrm{~b}$

$\begin{array}{lll}0.05 & 0.02 & 0.02\end{array}$

$4 \mathrm{~b}$

Agapostemon texanus

Agapostemon virescens 
Augochlorella aurata

Halictus confuses

$3 a$

Halictus tripartitus

$3 a$

Halictus rubicundus

$3 a$

Lasioglossum sp.

Ichneumonoidea

Cotesia sp.

Vespidae

Polistes dominula

Sphecidae

Ammophila sp.

Podalonia sp.

\section{Lepidoptera}

Crambidae

Loxostege sticticalis

Lycaenidae

Strymon melinus

Noctuidae

Estigmene acrea

Helicoverpa zea

Melanchra picta

Peridroma saucia

Spilosoma virginica

Spodoptera ornithogalli

Spodoptera exigua

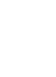

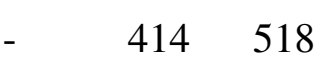

2

7

12

$1 \quad 5 \quad 4$

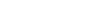


Trichoplusia ni

\begin{tabular}{ccc} 
& \multicolumn{2}{c}{ Trichoplusia ni } \\
& Nymphalidae & \\
& Tortricidae & Vanessa cardui \\
Mantodea & Mantidae & Grapholita delineana \\
Neuroptera & & Mantis religiosa \\
& & \\
& Chrysopidae & \\
& & Chrysopa,oculata \\
& & Chrysoperla nigricornis \\
& & Chrysoperla plorabunda
\end{tabular}

Hemerobiidae

Hemerobius sp.

\section{Odonata}

$\begin{array}{ll}\text { Libellulidae } & \\ & \text { Rhionaeschna multicolor } \\ & \text { Libellula sp. } \\ & \text { Sempetrum semicinctum }\end{array}$

Coenagrionidae

Enallagma spp.

Opiliones Phalangiidae

Phalangium opilio
$1 \mathrm{a}$

$1 \mathrm{a}$

$1 b, 1 c$

$\begin{array}{lll}0 & 1 & 3\end{array}$

$\begin{array}{lll}0 & 0.004 & 0.02\end{array}$

$4 a$

$\begin{array}{llllll}40 & 437 & 629 & 1.01 & 2.07 & 2.20\end{array}$

$4 \mathrm{a}$

$4 \mathrm{a}$

$4 a$

$\begin{array}{lll}0 & 0.02 & 0.007\end{array}$

$4 a$

$\begin{array}{llllll}1 & 4 & 1 & 0.02 & 0.02 & 0.003\end{array}$

$4 b$

$1 \quad 0 \quad 1$

$\begin{array}{lll}0.02 & 0 & 0.004\end{array}$

$4 b$

$4 \mathrm{~b}$

$6 \quad 17 \quad 24$

$\begin{array}{lll}0.15 & 0.08 & 0.09\end{array}$

$4 b$

$22 \quad 101 \quad 109$

$\begin{array}{lll}0.56 & 0.48 & 0.37\end{array}$ 


\title{
Orthoptera
}

Acrididae

$\begin{array}{lll}110 & 512 \quad 1022\end{array}$

$\begin{array}{lll}2.80 & 2.43 & 3.56\end{array}$

Aeoloplides turnbulli

$6 b$

Melanoplus bivattatus

1a

Melanoplus differentialis

1a

Melanoplus femurrubrum

$1 \mathrm{a}$

Melanoplus lakinus

Gryllidae

1

10

2

$\begin{array}{lll}0.02 & 0.05 & 0.007\end{array}$

Oecanthus sp.

1a

\section{Thysanoptera}

Aeolothripidae

Aeolothrips sp.

Thripidae

187

12121909

5.77

6.65

Frankliniella occidentalis

Thrips tabaci

TOTAL

3922

$21,01928,687$

100

100

100

\begin{abstract}
1 Key to hemp association: 1a Herbivore of hemp-defoliator; 1b Herbivore of hemp-stem borer, 1c Herbivore of hemp-chewer of buds/flowers and/or developing seed; 1d Herbivore of hemp - root feeder, chewing insect; 1e Leafminer - association with hemp undetermined; 2a Herbivore of hemp - fluid feeder of foliage; 2b Herbivore of hemp - fluid feeder of buds, flowers, and/or developing seeds; 2c Herbivore of hemp - root feeding, fluid feeder; 3a Hemp pollen feeder - bees; 3b Hemp pollen feeder, non-bees; 4a Natural enemy associated with hemp herbivore; $\mathbf{4 b}$ Natural enemy of insect/mite, uncertain association; 5a Scavenger/detritivore collected on hemp; 6a Incidental species on hemp, herbivore on other crops; 6b Incidental species on hemp; herbivore on weeds or unknown hosts; 7 Other.
\end{abstract}


Table 2.5 Proportion (percent) of arthropods to the order level, captured by sweep net during field surveys of hemp fields in eastern Colorado, Spring 2016-Fall 2018.

Order

Araneae

Coleoptera

Collembola

Dermaptera

Diptera

Hemiptera

Hymenoptera

Lepidoptera

Mantodea

Neuroptera

Odonata

Opiliones

Orthoptera

Thysanoptera
2016

3.26

13.21

0

0

28.57

40.08

7

0.5

0

1.00

0.02

0.56

2.82

5.00
2017

2.91

15.94

0

0

16.31

43.32

9.11

0.45

0.005

2.10

0.10

0.5

2.48

6.80
2018

3.36

16.00

0.02

0.01

17.66

40.33

8.80

0.40

0.01

2.20

0.91

0.40

3.60

7.18

\section{Arthropods found in hemp and their association with crop/feeding guilds}

The large number of kinds of arthropods present in hemp represent a variety of

associations with the plant. Those that feed on the plant, herbivores, include insects that chew on various parts of the plants (e.g., defoliators, leafminers, stem/bud borers) or insects and mites that suck fluids from leaves, flowers, or developing seeds. Some insects consume the pollen produced by hemp, a wind-pollinated plant, but these include species (e.g., many bees) that are important pollinators of other, insect-pollinated plants. Many of the arthropods found in hemp fields are natural enemies of arthropods, predators and parasitoids, many of which develop on the 
herbivores of hemp. Others are scavengers and detritivores that consume dead organic matter (including pollen) that collects on plants or is present on or within the soil. Also, surveys of a hemp field will find a great many incidental species, such as transient herbivores that develop on nearby crops or weeds that may enter a hemp temporarily to rest.

Approximately half (46\%) of the arthropods captured in sweep net sampling were natural enemies, mostly predators, feeding within hemp across all three years (Table 2.6). Many of these are large species, easily visible when entering the crop and quite amenable to collection with a sweep net, such as lady beetles and damsel bugs. The disproportionately high percentage of natural enemies caught in sweep net sampling is in part due to biases of this sampling method, which undersamples smaller species and those attached more firmly to the plant, including most herbivores.

Table 2.6 Proportion of the various arthropods collected in sweep net sampling hemp fields, arranged by their primary association with the plant. Sampling was conducted during Spring 2016-Fall of 2018, during surveys from eight counties in eastern Colorado.

\begin{tabular}{lrrr} 
Association with hemp & $\mathbf{2 0 1 6}$ & $\mathbf{2 0 1 7}$ & $\mathbf{2 0 1 8}$ \\
\hline Fluid Feeders of Foliage & 29.0 & 22.0 & 25.2 \\
Incidental Species & 15.7 & 17.0 & 17.5 \\
Defoliators & 3.3 & 3.0 & 4.0 \\
Natural Enemies & 44.0 & 49.0 & 46.2 \\
Pollen Feeders & 4.7 & 5.0 & 4.0 \\
Seed/Bud Feeders & 3.1 & 4.4 & 3.0
\end{tabular}

Various herbivores that feed on plant fluids from the foliage were the second largest group found in sweep net samples (25\%). This consisted mostly of various Hemiptera, particularly Aphididae (one species), Cicadellidae (14 species). Thrips, notably onion thrips, were also common residents of leaves. 
Herbivores of hemp that chew leaves (defoliators) or chewing and sucking insects that feed on developing buds or seeds were detected in smaller numbers, $3 \%$ and $4 \%$ of the total. Slightly higher numbers of insects captured in hemp (4.5\%) feed on hemp pollen.

The largest remaining group of arthropods collected (15\%) appear to have only an incidental association with the hemp crop. These include herbivores that develop on other crops or on weeds growing in close proximity to the sampled hemp. Some natural enemy species were frequently collected, such as damselflies and robber flies, which may temporarily rest on hemp plants.

\section{Herbivores of hemp}

Arthropods feeding on plants are said to be phytophagous or herbivorous. Fluid feeders meet their nutritional requirements through an entirely liquid diet, by feeding on plant sap. Twenty-five percent of arthropods collected over three years were found feeding on the phloem, flowers, and seeds of hemp. Phytophagous arthropods in hemp that feed on both phloem and mesophyll are considered pests of the crop.

\section{Acari}

Two species of plant feeding mites were found associated with hemp. Neither were collected by sweep net sampling but instead were sampled by field collection of leaves and subsequent examination using a microscope.

Tetranychidae. Twospotted spider mite, Tetranychus urticae (Koch) was frequently found present during indoor production phases of hemp. This species is highly polyphagous and a serious pest of vegetables (Capinera, 2001), fruit crops and many agronomic crops grown in Colorado, notably corn (Peairs, 2014). 
In hemp twospotted spider mite produces small white-grey to yellow leaf flecking (stippling) on leaves. In high populations leaf injuries show a generalized discoloration and may be killed (McPartland et al., 2000). Twospotted spider mite may also produce visible webbing when in high populations that can cover infested areas or even the entire plant.

Although this species is regularly observed on hemp being grown in greenhouses or protected sites (e.g., hoophouses) it has not been observed to damage field-grown hemp. This may be due to the presence of various predators (e.g., minute pirate bugs), adverse effects of weather or other factors that provide a high level of natural control of twospotted spider mite in field-grown hemp in Colorado.

Eriophyidae. Hemp russet mite, Aculops cannibicola (Farkas), is a minute species $(0.2 \mathrm{~mm})$ associated with leaves and developing flower buds of hemp. Cannabis spp. appear to be the only host for hemp russet mite. It has not been reported to develop on other plants, and in the course of these studies, attempts to establish hemp russet mite on other Cannabaceae family plants (hops, hackberry) were unsuccessful. Due to its small size, A. cannibicola was often present in plantings but not recognized or observed by producers. Due to their minute size, hemp russet mites are only capable of reaching into and damaging the surface layer of plant cells (epidermis), piercing the epidermis when feeding on the cell fluids. Damage by hemp russet mite is more subtle than that produced by twospotted spider mite and has not been well characterized. Heavily infested leaves often have a slight grayish or bronzed color change and some growers have reported that the mites have killed plants during indoor propagation. In some cultivars, a slight upward rolling of the leaf edge may occur; this symptom is not universally produced, and some hemp cultivars normally will produce similar leaf curling in the absence of the mite. More clearly damaging effects occur when hemp russet mite infests developing flower buds of 
cultivars grown for CBD production. High populations can reduce the size of flower buds and resin production, which can result in reduced yield of extractable cannabinoids (McPartland and Hillig, 2003).

Large numbers of hemp russet mite were observed on plants within enclosed production systems, but this species also reproduced well through the growing season in field plantings. In one field, (Site 6, Weld County) outdoor field populations were monitored weekly from June $6^{\text {th }}$, 2018 to September $20^{\text {th }}, 2018$. Leaves were collected from designated areas within the center of a field of hemp grown for CBD production. These leaves were then returned to the laboratory and then washed in a basin with ethanol, which removed the mites into the solution. These were later able to be counted under a microscope. These surveys found that hemp russet mite numbers increased steadily from about 50/leaf to about 450/leaf during the course of the survey (Figure 2.3). Mites were found in high numbers in all eleven samples indicating they persist for the entire growing season on outdoor grown hemp.

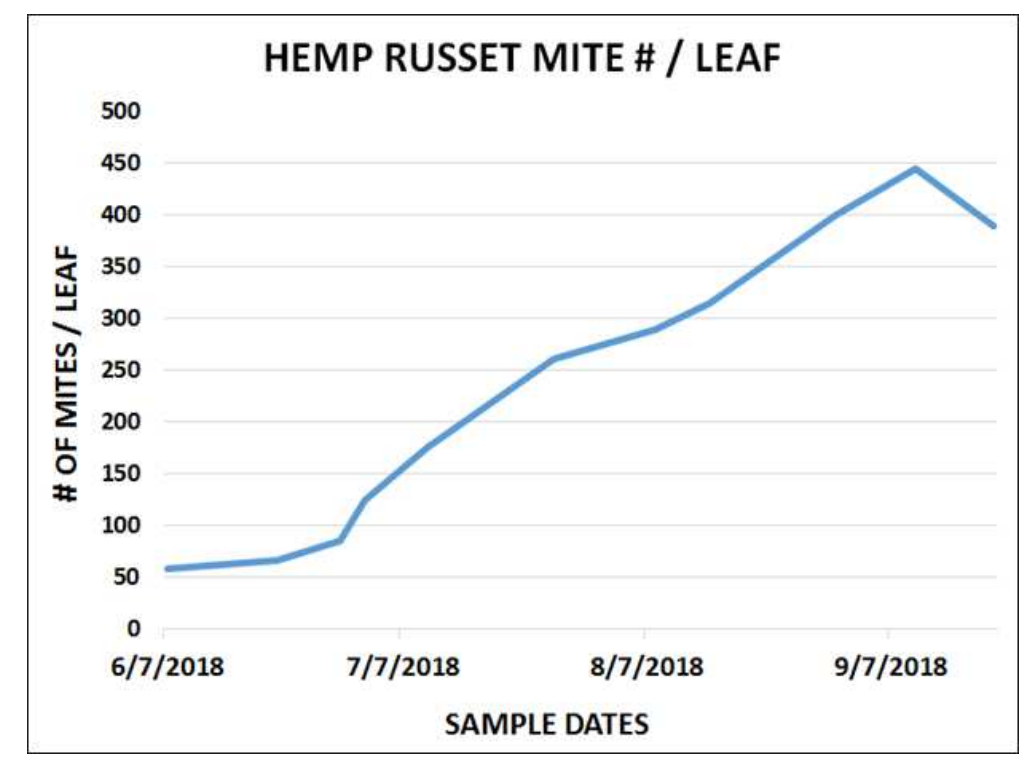

Sample Date Avg. Mite \#

\begin{tabular}{|c|c|}
\hline $6 / 7 / 2018$ & 57.7 \\
\hline $6 / 21 / 2018$ & 66.3 \\
\hline $6 / 29 / 2018$ & 84.5 \\
\hline $7 / 2 / 2018$ & 124.8 \\
\hline $7 / 10 / 2018$ & 175.2 \\
\hline $7 / 26 / 2018$ & 261.1 \\
\hline $8 / 8 / 2018$ & 289.8 \\
\hline $8 / 15 / 2018$ & 315.3 \\
\hline $8 / 31 / 2018$ & 400.78 \\
\hline $9 / 10 / 2018$ & 444.7 \\
\hline $9 / 20 / 2018$ & 388.8 \\
\hline
\end{tabular}

Figure 2.3 Hemp russet mite population tracked outdoors over the four-month season at Site 6 in Weld County, July 7 to September 20, 2018. 
Another small study addressed how hemp russet mites disperse themselves within an area. To investigate this, mites were monitored using glass slides lightly coated with petroleum jelly, which were affixed to posts and placed approximately $0,3 \mathrm{~m}$ above the crop canopy in both an indoor and an outdoor planting. These traps readily caught large numbers of mites, clearly documenting wind-blown dispersal by this species. Aerial dispersal is well known among eriophyid mites (Sabelius and Bruin, 1996).

The natural enemies, if any, which feed on hemp russet mite remain elusive. Minute pirate bugs were the only predatory species observed frequently in hemp russet mite-infested fields. However, although these insects are well known to feed on other insects common in hemp fields, such as aphids and thrips, the extent of predation by minute pirate bugs on hemp russet mite remains unclear. A second predatory species that more clearly appears to feed on hemp russet mite is the larva of the predatory cecidomyiid Feltiella acarisuga (Vallot), although it is not a common species found in hemp.

Phytoseiid mites, some of which are known to feed on eriophyid mites, were never recovered in any field collections of this study, and appear to be non-existent or very minor predators in hemp grown in eastern Colorado. Attempts were made using releases of phytoseiid mites acquired from commercial sources onto plants with high hemp russet mite populations. Two trials have been conducted, one involving release of large numbers of Amblysieus californicus in a greenhouse the second a smaller release of Amblysius swirskii. In both cases no evidence of reductions in hemp russet mite populations on plants was observed and the predators could not be recovered when examined a couple of weeks after the release. 


\section{Coleoptera}

Several families of Coleoptera are present in Colorado hemp fields but only a few, in the leaf beetle family Chrysomelidae, feed on the plant. Others are predators of arthropods, pollen feeders, scavengers/detritivores or incidental transients.

Chrysomelidae. The most commonly encountered leaf beetle in hemp, present in almost all fields is Diabrotica undecimpunctata howardi (L.), known both as the spotted cucumber beetle or the southern corn rootworm. This species has a very wide host range (Capinera (2001) and was reported in high numbers in marijuana grown in Mississippi by Lago and Standford (1989). Adults produce a minor amount of leaf chewing on hemp, which is often barely noticeable much less damaging to the crop. The larval stages of this insect develop on roots of various plants; it is unknown if hemp is a larval host. Although highly visible and regularly encountered this species appears to have little potential to ever significantly injure hemp.

Two types of flea beetles occur commonly in hemp; the western black flea beetle, Phyllotreta pusilla (Horn) and the palestriped flea beetle, Systena blanda (Melsheimer) (Lago and Stanford, 1989). Adults chew holes or pits into leaves and cotyledons of a broad range of young host plants characteristically described as "shotholes" (Bunn, 2015). The western black flea beetle is particularly damaging to Brassicaceae, including cultivated crops such as cabbage and canola, but has a broad host range (Doghairi et al., 2000; Chittenden and Marsh 1920). The palestriped flea beetle also has a wide host range that includes lettuce, potato, beans, potato and sunflowers. Both flea beetles, though present in hemp, cause only very small feeding wounds on leaves and never are present in high numbers. 


\section{Dermaptera}

Forficulidae. European earwig, Forficula auricularia L., is the only species of earwig found in Colorado and a common resident in yards and around gardens. The European earwig is omnivorous as it feeds on plant matter, particularly leaves and flowers. Yet, it is documented to feed on insects and may be very important as a predator of aphids, insect eggs, and small softbodied insects such as caterpillars. On hemp, feeding by European earwig produces minor leaf injuries. Earwigs only feed at night which would rarely attract attention of the grower. Significant infestations are unlikely in fields, but populations may develop well in and around greenhouses.

\section{Diptera}

The association of Diptera as herbivores of hemp remains unclear. There are dipteran leafminers that have been observed to develop in hemp leaves, but these have not been identified to even the genus level. Many other Diptera are present in hemp fields, but are involved in different feeding guilds, mostly as predators of arthropods or as scavengers/detritivores.

Agromyzidae. A high percentage of the insects caught in sweep net samples, between 2.5-6.5 percent, were leafminers of the family Agromyzidae. Larval leafminers develop within the interior removing mesophyll tissue, resulting in visible tunnelling of serpentine or blotch form Those observed in hemp primarily produced tunnels of meandering serpentine form.

No larvae developing in hemp were successfully reared to the adult stage. Adults of species in both the genera Agromyza and Liriomyza occurred commonly in sweep net sampling of hemp sweeps. Significant damage by leafminer larvae was never observed in hemp and these appear to be of negligible importance to the crops. It is also possible that many of the adults 
collected were incidental to hemp, developing on weeds within the hemp field or in adjacent crops.

\section{Hemiptera}

Hemiptera constituted the most species-rich order of insects in hemp collections, with representatives from 15 families with 36 genera identified during the course of the three year sampling period (Table 2.4). Hemipterans are found atop leaves, stems, buds, and flowers feeding and reproducing in hemp. Using their piercing-sucking mouthparts, all extract fluid from the plant but feeding site and feeding habit ultimately affect their potential to cause plant injury.

Aphididae. One dominant species of aphid was associated with hemp production in Colorado, the cannabis aphid, Phorodon cannabis (Passerini). This species, only recently recorded from North America (Cranshaw et al., 2018) was noted commonly in both indoor and outdoor production in all Colorado locations.

Cannabis aphid sucks fluids from the plant phloem, which they draw through their piercing-sucking mouthparts. In low numbers or when present only briefly, normally no visible damage is observable. However, in high populations that are sustained hemp may show wilting, yellowing and retarded growth. Such injuries are most commonly observed in indoor production. Outdoors there are a very large number of predators, along with a few parasitoids and fungal pathogens that will provide effective biological control.

Highest populations of cannabis aphid in fields were most often observed late in the season, sometimes being sustained into harvest. Large amounts of honeydew are produced during outbreaks, which may remain on plants if rainfall or irrigation does not wash it from the plants. 
Cannabis aphid is holocyclic on the crop and sexual forms (winged males, apterous oviparae) appear in early-mid September. Decreasing day length likely induces the production of egg producing females late in the season, which lay eggs that survive winter on crop debris remaining in fields after harvest. The eggs hatch in very late winter or early spring as apterous females (stem mother or fundatrix). Volunteer hemp seedlings that germinate the following spring and develop in very close proximity to crop debris with overwintering eggs support the early populations of cannabis aphid outdoors. This was confirmed to occur in 2018 field collections at two Weld County locations where large numbers of volunteer plants emerged in spring. Cannabis aphid can survive year round indoors, in continuous asexual reproduction, where lighting maintains long day length.

Cannabis aphid is reported to be monoecious on Cannabis spp (Müller and Ewald 1976) and transfers of this aphid to other Cannabaceae (hops, common hackberry) did not result in establishment and reproduction. Conversely, two aphids that have been reported to develop on Cannabis, green peach aphid, Myzus persicae (Sulzer), and hop aphid Phorodon humuli (Shrank), were unable to survive on hemp following transfers. It is speculated that these earlier reports may have resulted from misidentification of cannabis aphid.

The only other aphid found on hemp foliage was bean aphid, Aphis fabae (Scopoli), a species reported by McPartland et al., (2000). This was collected only a single time, from a spring collection of volunteer hemp in late spring 2018 collected from a Weld County field.

Rice root aphid, Rhopalosiphum rufiabdominale (Sasaki), develops on the roots of Cannabis plants and has only been observed on hemp grown in enclosed production. Hemp field sampling done during this study was not directed at identifying soil species, so the occurrence of rice root aphid in outdoor production is unknown. Rice root aphid has a generally round body 
form and have variable coloration. First and second instars appear salmon pink and can be found in stone wool used to root cuttings. As nymphs age, the colors darken and become mottled, ranging from shades of green to black. Wingless stages develop on roots and they survive on plants grown both in soil and in hydroponic culture. This aphid has a wide host range of plants but is most often associated with the roots of various grasses, including wheat, barley, and rice.

Cicadellidae. Leafhoppers were the most species-rich family of insects collected in hemp. To date, leafhoppers in 14 genera of leafhoppers have been identified so far from Colorado (Andrew Hicks, University of Colorado, personal communication, 2018). Ceratagallia uhleri (Van Duzee), was the most abundant leafhopper found in hemp samples, and is a species that was observed to breed commonly in outdoor grown hemp. Breeding was also confirmed by an Empoasca species, but immature stages were not found associated with most of the leafhoppers found in hemp suggesting that hemp is an incidental host for these species.

The leafhoppers observed in hemp in eastern Colorado appear to cause little, if any, significant plant injury. No visible symptoms on plants were associated with leafhoppers that feed on the phloem contents. The only visible damage, also insignificant, was a very modest amount of stippling produced by an Empoasca species, which feeds on mesophyll cells.

Although no serious pest issues were observed by leafhoppers during the survey period, it is important to note that in the 2019 season a leafhopper-vectored virus, beet curly top, produced a serious outbreak in hemp grown in western Colorado. Plants were also found infected in several locations in eastern Colorado during this season, but at much lower incidence. The vector of beet curly top is the beet leafhopper, Neoaliturus (=Circulifer) tenellus (Baker), an annual migrant into the state that originates from overwintering breeding sites present in more 
southwestern areas. Beet leafhopper has not been collected in hemp, suggesting that it will feed while passing through the crop but does not breed on hemp.

Membracidae. Two species of treehoppers were described from hemp field samples. Micrutalis calva (Say) and a Stictolobus sp. were found in sweep net samples from the canopy of hemp. Micrutalis calva is a small, polyphagous treehopper that is multivoltine (Deitz and Wallace, 2012). The adults have black pronota with pale tips and they appear yellow ventrally. Only adults of both species were collected from hemp so the ability of these treehoppers to reproduce on the crop is not established.

Cixiididae. A Melanoliarus species of cixid planthopper was commonly collected in sweep net samples of hemp. This is a fairly large planthopper, brown with hyaline and ornamented wings that extend well over the tip of its abdomen. Data on habits of Melanoliarus in North America are scarce but the immature stages of the genus are documented to be a subterranean feeder on roots of grasses (Mead and Kramer, 1981).

Pentatomidae. Several species of stink bugs are collected regularly in hemp. Most abundant and widespread is red-shouldered stink bug, Thyanta custator (Fabricius). Other plant feeding stink bugs present in the crop include the conchuela, Chlorochroa ligata (Say), and Chlorochroa uhleri (Stal). Eggs and nymphs were found in hemp, suggesting that these species successfully reproduce on the crop. These stink bugs are present prior to flowering so some feeding on foliage does occur, but they are most common in fields after flowering and are concentrated on flowers and developing seed but appear to cause little injury. These stink bugs have potential to cause problems with developing seed (abortion, seed pitting/distortion) but no damage by these insects has been observed and they are likely to be minor and possibly insignificant pests of seed production. 
Miridae. Plant bugs of the genus Lygus are extremely common on hemp and among the insects that are most consistently noted in casual observations of the crop. At least two species were regularly collected and found to breed on hemp, pale legume bug, Lygus elisus Van Duzee, and tarnished plant bug, L. lineolaris (Palisot de Beauvois). Lygus bugs are well documented to cause injuries to developing leaves, flowers and developing seeds and fruit. This suggests that they have potential to cause injury to hemp grown for seed. The only documented injury observed during this study associated with Lygus bug damage was some localized injury to new leaves, resulting in a minor twisting of the emerging leaves.

Rhopalidae. A very common insect found in hemp was hyaline grass bug, Liorhyssus hyalinus (Fabricius), particularly in crops grown for seed/fiber. This extremely polyphagous insect was found dominantly in Weld county breeding within the crop. Scentless plant bugs feed mainly on flowers, fruits, and seeds of their hosts. Thirteen species of host plants were recorded for $L$. hyalinus during the sampling of grasses, forbs, subshrubs, and shrubs in the western United States (Wheeler, 2016).

\section{Lepidoptera}

Several caterpillars have been noted to be associated with hemp. Most are defoliators, although none were documented to damage hemp to the point where yield is lowered. More injurious were species that tunneled into plants, particularly those that destroyed or damaged developing flower buds, and two key pests of the crop (corn earworm, Eurasian hemp borer) are lepidopterans. Lepidopterans were collected in low proportions within hemp. Part of this likely was sampling bias associated with use of a sweep net as caterpillars often clung to plants. Some caterpillars tunneled into stems or deeply into buds, making collection difficult, particularly 
those that tunneled into buds. Most observations of caterpillars in the crop resulted from direct observation.

Crambidae. Beet webworm, Loxostege sticticalis (L.), was a frequently collected caterpillar in hemp. This caterpillar is variable in color ranging from greenish to black; with a slender dark line longitudinally down its dorsal surface (Stehr, 1987). Damage by this species is more conspicuous than that of many other lepidopterans of hemp as they tie leaves together and feed within the leaf shelter constructed. Beet webworm has a wide host range and will feed on the leaves of carrot, celery, pea, potato, spinach, onions, sugarbeet (Peairs and Capinera, 2002). This caterpillar has records of pest damage in Larimer County on several crops (Gillette, 1905).

Erebidae. Two woollybear caterpillars were the most common defoliating caterpillars associated with hemp. Yellow woollybear, Spilosoma virginica (Fabricius), was most often observed, but saltmarsh caterpillar, Estigmene acrea (Drury), was also commonly found. Both have a dense, hairy appearance due to the presence of hairs known as verruca covering their integument (Stehr, 1987). Both were usually found in hemp late in the summer shortly before harvest.

Both saltmarsh caterpillar and yellow woollybear are generalist herbivores (Capinera, 2001). Adults lay eggs in the form of masses, and young stages after egg hatch will feed collectively for a brief period, producing noticeable skeletonizing to the leaf on which the eggs were laid. Later stage caterpillars scatter within the crop. These caterpillars appear to have negligible potential to cause significant injury to hemp as injuries are confined to leaves, occur late in the season, and populations in hemp are low. 
Lycaenidae. Larvae of the cotton square borer (gray hairstreak), Strymon melinus (Hübner), have been collected from hemp and observed to feed on foliage. Hemp is a minor host of this species and produces insignificant injury to the crop.

Noctuidae. Several species of climbing cutworms can be found on hemp producing minor injuries to foliage. These include beet armyworm, Spodoptera exigua (Hübner), variegated cutworm, Peridroma saucia (Hübner), and the yellowstriped armyworm, Spodoptera ornithigalli (Guenée). The latter species was the most regularly sampled species, present after flowering, but none were ever found abundant in hemp crops.

Zebra caterpillar, Melanchra picta (Harris), was found more consistently than the other defoliating noctuids at sites in Larimer and Weld counties and was present throughout the 2018 growing season. This caterpillar can be distinguished from other noctuids from two yellow stripes running longitudinally down the integument. This insect can be found defoliating a variety of broadleaf field and vegetable crops, ornamental trees and many other flowering plants (Peairs and Capinera, 2002). In hemp it appears to particularly favor male flowers, and was not observed to cause extensive injury.

Corn earworm, Helicoverpa zea Boddie, was not observed to feed on foliage but instead feeds on the developing female flower buds, which are the primary part of the plant grown for production of CBD hemp. This species, also known as the tomato fruitworm and the bollworm, is also common in cultivated crops including beans, corn, cotton, lettuce, peppers, tomatoes, soybeans, and sorghum (Cook and Weinzierl, 2004). Corn is a common host in Colorado, attractive to the egg laying adults during the period it is silking and kernels beginning 
to develop. After corn becomes more mature and unsuitable as a host, hemp fields maturing late in the season become attractive oviposition sites.

Serious crop damage occurs when caterpillars burrow into the maturing female flower buds. Large areas of tissue are directly consumed and further injury can occur when areas of buds are clipped, causing sections to die or even drop from plants. Conspicuous amounts of frass are also deposited within the damaged buds, further damaging quality. Sporadic outbreaks of corn earworm have recently occurred in the Arkansas Valley, in 2016, 2018 and 2019. The corn earworm is the most significant insect pest observed in CBD hemp in Virginia, Colorado, and Kentucky (Britt et al., 2019; Cranshaw et al., 2019) and this species occurs throughout the continental United States. Potential damage to fiber or seed producing cultivars is much less likely than to CBD cultivars that produce large buds of unfertilized flowers. To date, corn earworm has demonstrated the greatest potential to damage hemp among all arthropods associated with the crop in the United States.

Nymphalidae. Painted lady, Vanessa cardui (L.) is a highly polyphagous species that has been found to feed on many crops including corn, alfalfa, beans, sunflowers, and soybeans (Williams, 1970). Many non-crop plants also host this insect, particularly thistles and certain mallow family plants. Painted lady caterpillars were observed feeding on hemp foliage, as occurs with other hosts, the caterpillars feeding on hemp tie together leaves to make a silken shelter, tending to make the presence of this insect more conspicuous than other defoliators found in the crop. However, despite this insect being present in high numbers in eastern Colorado during the years of this survey these caterpillars were rarely found in hemp and it appears to be a minor insect.

Tortricidae. Eurasian hemp borer, Grapholita delineana Walker, has been found to be widespread in eastern Colorado, and has been caused significant injury to fields in Yuma 
County. Eurasian hemp borer has been historically recorded from the Midwest (Miller, 1982), associated with feral hemp, and the Colorado collections of this species extend by at least 500 miles the known range of this insect within the United States.

Eurasian hemp borer spends almost its entire larval life within the hemp plant. Immediately after egg hatch larvae tunnel into petioles, stems and stalks (McPartland, 2002). At least three generations appear to be produced in Colorado, with damage by early generations confined to stems/stalks, an injury that can produce some plant stunting. Most serious injuries occur in the late season generation, when developing flower buds are present. Larval tunneling at this time can kill parts of or often the entire flower bud. This is particularly damaging to CBD cultivars, but Eurasian hemp borer is also a potentially serious pest of hemp grown for seed. Kryachko et al., (1965) found G. delineana destroying 80\% of flowering tops of seed cultivars.

\section{Orthoptera}

Acrididae. The most dominant defoliators of the crop (80\%) collected in this survey were grasshoppers. Grasshoppers were observed to cause two kinds of injury to plants. Leaf feeding (defoliation) was most common and noticeable, but some species also caused serious damage when chewing on stems and stalks. These produced girdling wounds that often resulting in dieback or breakage beyond the wound site.

At least four species of grasshoppers have been confirmed to feed on hemp: differential grasshopper, Melanoplus differentialis (Thomas); twostriped grasshopper, M. bivittatus (Say); redlegged grasshopper, M. femurrubrum (DeGeer); and M. lakinus (Scudder). There were documented outbreaks in several counties with significant damage reported in 2016, 2017, and 2019. Grasshopper species vary in abundance depending on the soil, vegetation, topography, and use of a habitat. The densities of grasshopper species inhabiting a farm site may change with 
time because of effects of weather, parasites, disease, or insecticidal treatments (Pfadt, 1994).

Grasshopper populations increase where there are warm temperatures and low rainfall, particularly during summer, and numbers varying considerably both within and between seasons (Capinera and Sechrist, 1982). All four of the grasshoppers found in hemp are generalist herbivores.

Most damage to hemp was observed by twostriped grasshopper, the species most associated with damage to stems and stalks. This grasshopper consumes several diverse types of host plants (Bailey and Mukerji, 1976). Several types of crops report economic losses from the twostriped grasshopper (Olfert, 1986; Olfert and Slinkard, 1999). A hemp grower in Larimer County estimated losing half a million dollars in their CBD production during 2019 due to heavy infestations of twostriped grasshopper. Populations of this grasshopper moved into the hemp crop once surrounding fields dried under the late summer sun.

Differential grasshopper was also very regularly found in hemp. This species can be found on in agricultural landscapes across the Front Range of Colorado. They are documented to be abundant and destructive in Colorado's lower elevations (Bruner, 1897; Alexander, 1941). Like twostriped grasshopper, they will often roost on stalks during the night and may cause extensive damage to stems and stalks.

Redlegged grasshopper is the most commonly encountered species found associated with areas of open weeds and cultivated habitats (Bruner, 1897; Hebard 1928, Alexander 1941). It has been reported to be especially abundant in cultivated land that has been deserted for several years. This species readily feeds on hemp leaves but was not noted to damage stems and stalks. 
Melanoplus lakinus (Lakins grasshopper) feeds primarily on plants of the Chenopodiaceae plant family; sugarbeet, and Russian thistle (Gillette, 1904; Mulkern, et al., 1969) This species is one more common from the Great Plains but was not found abundantly from our surveys. M. lakinus is not an economically important species in grassland habitats and is considered an incidental species in industrial hemp fields (Hantsbarger, 1979; Hebard, 1929; Mulkern et al., 1969; Cranshaw et al., 2019).

Many grasshoppers present in eastern Colorado, including those found in hemp fields, primarily feed on weeds or non-economically important plants. An example is Russianthistle grasshopper, Aeoloplides turnbulli (Thomas), a species that specializes in plants in the family Amaranthaceae, including Gardner saltbush and many common weeds such as Russian thistle, kochia, and lambsquarters. It was observed in hemp fields, but restricted its feeding to kochia.

Gryllidae. Tree crickets (Oecanthus sp.) (Gryllidae), were also collected in hemp fields. Adults and immature stages have been recorded to cause very minor leaf feeding and have not been documented in high abundance. Oecanthus species are omnivorous feeding upon leaves, flowers, fruit, fungi and such small insects as aphids, and scales (Ball et al., 1942). Oviposition and slight chewing habits are damaging to plants, but the harm is offset by the good done by the tree crickets feeding on pest species of arthropods (Ball et al., 1942; Blatchley, 1920).

\section{Thysanoptera}

Thripidae. Thrips comprised approximately one fourth the insects that feed on plant fluids which were collected in surveys. Dominant, perhaps exclusive, on foliage was onion thrips, Thrips tabaci (Lindemann), a ubiquitous species of wide host range found throughout North America (van Rijn et al., 1995; de Jager et al., 1997; Theunissen and Schelling 1997; van Dijken 
et al., 1994; Kirk and Terry 2003). When pollen is produced, the western flower thrips, Frankliniella occidentalis (Pergrande), was found commonly on hemp. Sites that we surveyed with flowering hemp seemed to have much higher proportions of thrips compared to sites that were grown for CBD production. Thrips cause stippling injury when they feed on the leaf tissues. When populations are sustained at high levels cumulative injuries can cause extensive leaf scarring in hemp. However, in outdoor plantings of hemp not significant damage was observed.

\section{Pollen feeders in hemp}

Hemp is a wind pollinated plant and enormous amounts of pollen can be produced in hemp crops when male flowers are present. Pollen feeding species became common in hemp fields grown for seed, fiber, or grain. When male plants began to flower in late summer, many pollen feeders became advantageous and were found buzzing when industrial hemp blooms. Hemp does not produce floral nectaries, meaning it is not possible for bees to gather nectar for honey production. However, they do seek out this available resource considering it is rich in protein. A single hemp flower can produce approximately three hundred fifty thousand pollen grains (Faegri et al., 1989), and there are hundreds of flowers on mature male plants.

Bees (Apidae, Halictidae). Seventy percent of the pollen-feeding insects collected from hemp fields were bees in the families Apidae and Halictidae, including representatives of 13 genera. Honey bee, Apis mellifera L., was the species found in highest proportion out of any sampled pollen feeder found in hemp fields during flowering.

Although the honey bee is an introduced species, Colorado hosts a very wide diversity native bee species, with almost one thousand species represented across the state (Scott et al., 2011). Twenty-three different genera of digger bees, sweat bees, and bumble bees were 
documented in flowering hemp fields using blue vane traps (O'Brien and Arathi H. S., 2019). Various long-horned bees such as Melissodes agilis (Cresson) and sweat bees (Agapostemon. Halictus, Lasioglossum) were found in the highest proportion over the three-year sample.

In agricultural areas of Colorado, there are often limited sources of pollen available in late summer when hemp is in bloom (typically August and early September in Colorado). In these setting pollen from hemp plants may provide a valuable resource for many species of bees, both native and non-native (e.g., honey bee). Pollen collection does not occur, of course, in hemp where male flowers are absent, typical of crops grown for CBD. However, there were personal observations of honey bees on CBD crops, possibly collecting resins for propolis production.

Non-bee pollen feeders. Flowering hemp is a significant source of pollen for other kinds of pollen feeders during late summer. Thirty percent of pollen feeding species collected in hemp were not bees, with most being beetles. Five families of beetles were found commonly when the high pollen resources are made available by the hemp plant.

Anthicidae. Ant-like flower beetles are small rusty colored beetles that were occasionally found within sweep samples from flowering hemp. The common genera documented are Anthicus and Notoxus. These two genera can be separated from each other from characteristics on the pronotum, dorsally. Notoxus has a hornlike projection off the thorax that extends over the head and Anthicus lacks any pronotal horn.

Melyridae. Malachius aeneus L. was documented in hemp grown primarily from Larimer and Weld counties. Soft winged flower beetles are common at flowers considering many adults consume pollen as their main protein source. This species is dominantly red with accents of metallic blue-grey color and is also very commonly associated with leafy spurge. 
Morellidae. Tumbling flower beetles are wedge-shaped beetles that can be distinguished by the distinctive "pintail" and hunched dorsal side. Two genera of tumbling flower beetles occur in hemp, Mordella sp. and Mordellistena sp., which are predominantly dark grey or black and brown or black, respectively (Cranshaw and Shetlar, 2018). Mordellistena sp. are mentioned in (McPartland, 2000) to be associated with hemp as a stalk borer. Stalk boring of hemp by mordellids was not observed in this study.

Phalacridae. Shining flower beetles (1-3 mm) where individuals are highly specialized feeders on ascomycetes hyphal spores. Olibrus visits flowering plants in the adult form, known to consume pollen (Gimmel, 2013). Olibrus adults were collected all three years in hemp fields when available pollen was present in late summer.

\section{Natural enemies in hemp}

The largest single group of arthropods collected during sweep net sampling of hemp fields in this study, comprising forty-seven percent of collections, were natural enemies of arthropods, predators and parasitoids. Natural enemies were collected from ten orders, representing thirty-seven arthropod families (Table 2.4). This suggests that there exists a robust complex of natural enemies of hemp pest arthropods and their identification by growers will be very important when assessing potential pest problems.

\section{Acari}

Phytoseiidae. In this study no predatory mites were ever found in field samples. Attempts were made to introduce the species Amblyseius andersoni both outdoors and in hemp confined in a greenhouse, but the mite was not recovered from leaf samples. The absence of predatory mites as a part of the natural enemy complex in Colorado may, in part, be due to low humidity. Most commercially available phytoseiid mites, including A. andersoni, are recommended for sites 
where relative humidity is 50-70 percent or higher, conditions that are rarely sustained in most areas of eastern Colorado (Gomez-Moya et al., 2018).

\section{Araneae}

Spiders are natural enemies of insects and other arthropods and are often very important in managing pests in agricultural crops. Spiders contribute to suppressing several hemp insects pests including various caterpillars, young stages of stink bugs, and Lygus bugs, aphids, and other insects. In hemp, arachnids had representatives collected from 2 orders and 8 families (Table 2.4). Spiders and opiliones made up $8.7 \%$ of the natural enemies sampled from hemp.

Araneidae. The orbweaver spiders are the largest and most conspicuous of the spiders found in hemp, constructing large concentrically patterned sticky orb webs. The most commonly collected were from the genera Neoscona and Araneus. Occasionally seen, but uncommonly collected, was the large whitebacked (banded) garden spider Argiope trifasciata. Orb weavers found in hemp spin sticky webs to capture flying prey items such as flies, bees, wasps, and leafhoppers. The large webs of the banded garden spider allow them to also capture grasshoppers.

Thomisidae. The most commonly collected spider in hemp fields was the crab spider Mecaphesa celer, sometimes known as the "swift crab spider". This is an ambush hunter and it was common to observe these spiders awaiting motionless on leaves or near flowers for passing insect prey.). Mecaphesa is the dominant group of Misumenini in all parts of the Nearctic region (Lehtinen and Marusik, 2008).

Philodromidae. The running crab spiders, mostly in the genus Philodromus, are encountered commonly in the field. This species is associated with both trees and forbs (Cokendolpher et al., 1979). Most are dull colored from brown, gray, yellowish or mottled with a leaf-like cardiac 
mark on the anterior dorsal abdomen. None of the species build webs, but they do use silk for draglines and egg sacs. Their second legs are usually the longer of the four pairs of walking legs. A Tibellus species was also captured in sweep net samping.

Salticidae. More species of jumping spiders were recovered from hemp fields than any other spider family. Particularly abundant were Phiddipus audax (Hentz) and Phiddipus clarus (Keyserling). Other common salticids are in the genera Eris, Pelegrina, and Siticus that are sighted on leaves, stems or flowers. Jumping spiders are very active hunters with excellent vision. Phidippus audax is a known predator of numerous major crop pests, including boll weevil, spotted cucumber beetle, and other beetles; fall webworm, and other moths, lygus bugs, stink bugs, leafhoppers, midges, mosquitoes, and other flies (Bailey and Chada, 1968; Clark and Glick, 1961; Kagan, 1943; Whitcomb et al., 1963; Young, 1989a \& 1989b).

Tetragnathidae. The most common spider found making webs in hemp was a longjawed spider of the genus Tetragnatha. These spiders also make concentrically patterned sticky webs designed to capture small flying insects.

\section{Opiliones}

Phalangidae. Daddy long legs, are a cosmopolitan group of arthropods with many species associated with crops (Sankey and Savory, 1974; Pinto-Da-Rocha et al., 2007; Drummond et al., 2010). In Colorado hemp the non-native species Phalangium opilio (L) was often collected. This is a generalist predator found in many agricultural landscapes actively hunting prey (Drummond et al., 2010). Two studies recognized that $P$. opilio fed on corn earworm eggs in soybean fields (Newton and Yeargan, 2001; Pfannenstiel and Yeargan, 2002). 


\section{Coleoptera}

Various predatory beetles were among the most conspicuous of the insect predators found in hemp. Four families of beetles were represented in collections, predominantly Coccinellide and Melyridae. (Table 2.4).

Coccinellidae. Perhaps the most consistently observed insect one finds, throughout the growing season, when entering a hemp field in eastern Colorado is the convergent lady beetle, Hippodamia convergens (Guérin-Méneville). It is a generalist predator, but reproduction in the crop appeared associated with the presence of cannabis aphid. When high populations of aphids were present several other species of lady beetles were present, particularly the multicolored Asian lady beetle, Harmonia axyridis (Pallas) and sevenspotted lady beetle, Coccinella septempunctata L. These species, both non-natives to North America, were also observed to breed on cannabis aphid-infested plans. Less common, but regularly collected, species included Coccinella novemnotata (Herbst), Coccinella transversogutta (Faldermann), Hippodamia parenthesis (Say), and Olla v-nigrum (Mulsant).

Melyridae. Twospotted collops beetle, Collops bipunctatus (Say), is a generalist predator common to Colorado (Fall, 1912). In hemp it is likely feeding on aphids, nymphs of Lygus bugs, early instars of caterpillars and other small insects associated with the crop. Pollen may supplement the diet of the adult beetles, but they are considered generalist predators as they consume a great diversity of insect fauna. Larval stages occur in soil and develop as predators of soil-dwelling insects. Both male and female adult insects are generally similar in appearance and size, but can be differentiated by the antennae, which are straight with the females and are distinctly enlarged at the base with the males. 
Lampyridae. Pyrogyga minuta and other Pyropyga spp. are small, non-luminescent fireflies that were sporadically spotted in the hemp canopy, often as mating pairs. They share the same general appearance of the cantharid also commonly found in hemp, but have the head area more completely obscured by the pronotum when viewed from above. Pyroyga often have the pronotum completely bordered with black (Green, 1961). Larval stages of lampyrids are predators arthropods in soil, but the adults are falsely known as predators.

Cantharidae. A soldier beetle, tentatively identified in the genus Cantharis, was found in hemp field samples. Adults of this genus are predaceous on other insects.

\section{Diptera}

Over twenty percent of natural enemies recorded were in the order Diptera. Seven families of predacious dipterans were described with representatives from eight genera (Table 2.4).

Cecidomyiidae. Larvae of two predaceous gall midges are present in hemp. Most common is Aphidoletes aphidimyza (Rondani), the aphid predator midge, which would be found late in the season feeding within dense colonies of cannabis aphid. Aphid predator midge overwinters in subterranean cocoons followed by pupating in spring. Adults emerge and reproduce the same day and the mated females begin the search for aphids. Their elongate eggs are laid during the first few days after emergence near colonies of aphids (Markkula et al.,1979).

A second predatory midge, Feltiella acarisuga (Vallot). was discovered during Berlese funnel sampling of foliage infested with hemp russet mite. This is a species well known to feed on many species of spider mites (Mo and Liu, 2007) and it has been incorporated into integrated pest management programs (Gillespie et al., 1998). Their presence on hemp leaves infested with 
hemp russet mite, where spider mites were absent, is suggestive that this species will feed and develop on hemp russet mite.

Chloropidae. The predatory chloropid, Thaumatomyia glabra (Meigan), is one of the most abundant predators collected in this survey. Thaumatomyia. glabra is found to be predaceous on several kinds of root aphids (Sabrosky, 1935; Dunn, 1960; Cole and Schlinger, 1969; Narchuk, 2000). T. glabra was prolific in both greenhouse and field samples over the three-year sampling period. Larvae of $T$. glabra could be surviving feeding on root infesting aphids, including rice root aphid or sugarbeet root aphid, in hemp fields developing on hemp or weeds that support these insects.

Dolichopodidae. Long-legged flies of the genus Condylostylus (Bigot), were often observed sunning themselves on hemp leaves. Adults are predaceous on many kinds of small insects that may occur in hemp (aphids, leafhoppers, small midges). Larvae of most long-legged flies develop as predators in soil and feed on soil-dwelling arthropods. Over thirty species are found north of Mexico, but only three west of Rocky Mountains (Pollet et al., 2004). Multiple species occur in hemp, but several were not identified past the family level.

Hybotidae. Hybotid dance flies, Platypalpus (Macquart), are small flies often found on hemp foliage. Adults are predaceous on small insects, and have large mesothoracic legs modified to hold prey while the fly feeds. Several species occur in the hemp crop that were not identified past the family level.

Pipunculidae. The big-headed flies, were found occasionally in hemp. These small, black flies are difficult to identify but the entire family is predaceous group found mainly feeding on leafhoppers and treehoppers (Skevington and Marshall, 1998). Pipunculidae members are 
differentiated from syrphids by large compound eyes that occupy most of their hemispherical head, distinctive wing venation (no vena spuria, open discal cell) (Skevington and Yeates, 2001). Syrphidae. Adult flower flies were commonly collected in hemp, particularly in association with plants supporting high populations of cannabis aphids. Flower fly larvae of at least four species were observed feeding on cannabis aphids in hemp fields and in high tunnels, but identification to the species level was not made since they were not successfully reared to the adult stage.

In addition to the predatory syrphids, the drone fly, Eristalis tenax (L.) was collected. Larvae of this species, known as "rattaailed maggots" are saprophagous, feeding on decaying organic matter in very moist soils. Periodically ponded water in wheel tracks of irrigation equipment provide one habitat well suited for development of this species in some hemp fields.

\section{Hemiptera}

Thirty-three percent of natural enemies were identified to belong to the order Hemiptera representing 7 families. Predaceous hemipteran use their rostrum to pierce the body of their prey and feed on the host blood.

Anthocoridae. The minute pirate bug Orius insidiosus (Say) was a common predator found hunting small arthropods in hemp fields. Immature stages (nymphs) and adults feed on a variety of small prey including thrips, spider mites, insect eggs, aphids, and small caterpillars. They were also found often on plants were the most abundant small arthropod was hemp russet mite, so it is possible that it may taken russet mites as prey. Orius insidiosus is, by far, the most common anthocorid in the eastern and central part of the continent (Slater et al., 1978). It is possible that Orius tristicolor (White) also occurs in hemp considering it is the most common species occurring in western states (Schaefer, 1991), but this was not identified from samples. 
Berytidae. A Jalysus sp. stilt bug was occasionally captured in sweep net samples Stilt bugs develop primarily by feeding on small insects and insect eggs. They also drink plant sap with their piercing-sucking mouthparts, but such feeding would not produce any effects on hemp plants.

Geocoridae. The big-eyed bug Geocoris punctipes (Say) is a generalist predator of insects and is common in many cultivated crops grown in Colorado. These predators can also be commonly found on weeds and sparse grasses. G. punctipes is commonly associated with weedy Chenopodium species (Slater and Baranowski, 1990). In hemp, bigeyed bugs are likely feeding on aphids, nymphs of Lygus bugs, leafhoppers, young caterpillars and other small insects associated with the crop. Prey items can also include eggs, caterpillars, leafhoppers, mites, thrips, whiteflies, and aphids. Big-eyed bugs are generally grayish with a dark marking on the center of their dorsal side. Nymphs are smaller, lack wings, and are found in hemp indicating this species does reproduce on the crop.

Miridae. Ragweed plant bug, Chlamydatus associatus (Uhler), constituted a very high percentage of the insects collected in sweep net samples, between 4.4-6.5\% of the total. This is considered to be a predatory species, and its common occurrence in hemp suggests it could be a signficant predator of small arthropods. Nymphs were occasionally collected so it is known to breed within outdoor hemp fields.

Although known as the "ragweed plant bug" it is a generalist predator, feeding on small insects such as aphids, thrips, and plant bug nymphs. C. associatus is documented to breed on weedy Ambrosia sp., (Knight, 1923, 1941; Blatchley, 1926; Schuh and Schwartz 2005), which is a native weed in the High Plains of Colorado. Ragweed plant bugs are also collected on a much wider range of plants, aligning with the list produced by Kelton (1965). 
Nabidae. The damsel bug Nabis alternatus (Parshley) is an extremely common predator found in hemp fields, present throughout the season. This is a generalist predator, which will attack many insect species (Harris, 1928; Knowlton, 1943, 1944). In hemp, Nabis alternatus is likely feeding on aphids, nymphs of Lygus bugs, leafhoppers, young stages of caterpillars and other small arthropods. Reproduction within the crop does occur and multiple generations are seen each year in Colorado.

Pentatomidae. The predacous twospotted stink bug, Perillus bioculatus (Fabricius) was collected in Weld County on rare occasion. This is primarily a predator of beetle larvae, and its collection could have been due to the presence of incidental hosts feeding on weeds near the crop, such as Colorado potato beetle on solanaceous weeds (O'Donnell and Schaefer, 2018; McPherson 1982).

Reduviidae. Spined assassin bug, Sinea diadema (Fabricius), is a medium sized, grayishbrown, spiny reduviid that was fairly commonly found in hemp fields. It feeds on wide variety of insects, but has been reported to favor small bugs (e.g., Lygus bugs) and beetles. Eggs and nymphs of this species have been found in hemp field samples indicating it does breed within the crop. Migration into hemp by adults from off-field sites occurs in early summer and it does produce two generations a year (Readio, 1924).

Other assassin bugs found in hemp fields are Zelus tetrancanthus Stal and the ambush bug Phymata americana Melin. The latter moves into flowering hemp to prey on flying insects present in fields late in the season. This insect does not reproduce within the hemp crop.

\section{Hymenoptera}

Twelve percent of natural enemies belonged to the order Hymenoptera over the threeyear sample. The great majority of these were parasitoids, most within either the superfamily 
Chalcidoidea or Ichneumonoidea. These are very difficult to identify and further identification to a family or genus was not attempted.

During this project attention was given to identify specific parasitoids that were observed to develop with key pests of the crop. Cannabis aphid was confirmed to be host for at least two species of parasitoid wasps in the family Braconidae, Aphidius ervi and Aphidius colemani, using characteristics from Rakhshani et al., (2012). Both of these are commercially available species as biological control agents and these identifications provide the first records of cannabis aphid as a host.

Some predaceous Hymenoptera were also recovered, but in low numbers. These including the social species Polistes dominula (European paper wasp), a well known predator of many insects, particularly of caterpillars. A small number of hunting wasps (Sphecidae family) were also collected in sweep net samples.

\section{Mantodea}

Mantidae European mantid, Mantis religiosa L., is a non-native generalist predator of insects. In hemp, younger mantids appear in late spring/early summer and would likely prey on leafhoppers and small flies. As the mantids mature larger insects such as grasshoppers, larger flies, bees, and beetles are feasted upon. Both green and brownish forms occur in Colorado where all mantids are univoltine. The overwintering stage is an egg case referred to as an ootheca. Egg cases are attached to rocks, stems and other solid surfaces during late summer and early fall by adult females.

\section{Neuroptera}

Four percent of natural enemies collected from hemp were from the order Neuroptera.

Chrysopidae. Three species of green lacewings were collected as adults and larvae from 
industrial hemp: Chrysopa oculata (Say), Chrysoperla plorabunda (Fitch), and Chrysoperla nigricornis (Burmeister). Green lacewings, adults are green with golden eyes belonging to the family, Crysopidae. Adults of Chrysopa may be predacious on aphids but Chrysoperla adults consume nectar or honeydew. Lacewing habitat includes grasses, forbs, trees, shrubs, and weeds.

Chrysopid larvae use their sickle shaped mandibles to predate on other insects, especially aphids (Stehr, 1987). Larvae "aphid lions' are 2-10mm in length and will consume larger insects, insect eggs, spider mites, and pupae. Larvae have been noted to consume up to 400 aphids during their two-week larval cycle (Hill, 1994). Chrysopid eggs are laid on stalks to prevent the cannibalistic larvae from consuming one another following hatching.

Hemerobiidae. The brown lacewings are related to the green lacewings with generally similar biology and behavior (Cranshaw and Shetlar, 2018). Brown lacewings from the genus Hemerobius were collected occasionally in Weld County but are typically associated with woodlands. Brown lacewing females attach eggs directly to leaves unlike their sister group, Chrysopidae, which lay eggs on stalks. Wings are rounded, brown, with membrane covered with small hairs.

\section{Thysanoptera}

Aeolothripidae. Predatory thrips of the genus Aeolothrips "banded thrips", were observed infrequently in hemp samples. Banded thrips feed on a mixed diet of pollen and the larvae of other thrips making hemp with pollen a suitable site for this predator (Mound, 2005, Trdan et al., 2005) and they were most often collected from flowering hemp. Approximately one hundred species have been recorded within Aeolothrips and the majority spend most of their lives in flowers, feeding on small arthropods and pollen (Patrzich and Klumpp, 1991; Mound and Kibby 1998; Yee et al., 2001). 


\section{Common incidental species found in hemp}

Incidental insects are those considered to be transients, where some may take advantage of the habitat provided by the hemp crop. Many species are found to be associated with commonly observed agricultural weeds (Table 2.2). Fifteen percent of arthropods found in hemp samples were transients that were not associated with hemp directly. These insects may hunt or live within the hemp canopy, but do not feed or reproduce on hemp directly.

\section{Coleoptera}

Coleopterans were common visitors in Colorado hemp fields, but few species threaten the crop. Many beetles found on hemp farms are also commonly known from agricultural fields as pests. The majority of the beetles found on hemp farms are not found to be associated with hemp for reproduction or nutrition and were considered incidental.

Chrysomelidae Some of the species of flea beetles found in hemp appear to be incidental species that do not feed on the crop. An Altica sp. was occasionally collected in sweep samples. An unknown Disonycha species was spotted occasionally in hemp but was not observed to feed on the plant and is considered incidental. Both of these genera include several common species that can be found on a wide variety of plants.

The diabroticine beetles western corn rootworm, Diabrotica virgifera virgifera

LeConte, and, less commonly, striped cucumber beetle, Acalymma vittatum F., were frequently collected in hemp fields. Neither of these species feed on hemp, but develop in nearby crops, corn and cucucubits, respectively. 
Several tortoise beetles were also found in sweep net collections. The mottled tortoise beetle, Deloyala guttata (Olivier), is most common followed by the golden tortoise beetle, Charidotella sexpunctata (Fabricius). A somewhat larger species of tortoise beetle is the Argus tortoise beetle, Chelymorpha cassidea (Fabricius), and was found abundantly only in Weld county. The Argus tortoise beetle is also associated with bindweed and morning glory. This is the largest tortoise beetle in Colorado and it can be recognized from its orange with dark spotting. All of these develop on morningglory family plants (Convolvulaceae), such as field bindweed, a common weed in many hemp fields. Colorado potato beetle, Leptinotarsa decemlineata L., occasionally was seen in hemp fields where buffalo bur (Solanum rostratum) was present.

Scarabeaidae. Bumble flower beetle, Euphoria inda L., is a hairy scarab that is present in mid to late-summer. They are scavengers, most often observed at flowers (e.g., daylilies, large thistles) or visiting fermenting fruit. In hemp they have been observed to visit frothy ooze resulting from bacterial infection of wounded stalks; fermenting pollen may also be eaten.

\section{Hymenoptera}

Argidae. Schizocerella pilicornis (Homgren) is found in hemp fields only because common purslane (Portuacha oleracea) is abundant in cultivated fields in Colorado. Purslane is this insect's only host and is not associated directly with hemp. This non-stinging wasp is often present in fields with purslane growing between the hemp canopy. Larvae develop as blotch leafminers within leaves of the purslane plant and would only be seen if exposed manually from within the leaf. This wasp rests on the foliage of all kinds of plants and adults only live a single day to reproduce. 


\section{Hemiptera}

Lygaeidae. Small milkweed bug, Lygaeus kalmii (Stal), is an insect that is very commonly encountered in all manner of sites in eastern Colorado - agricultural fields, meadows, and gardens. Adults feed primarily on flowers and developing seeds of various herbaceous plants, including many common weeds, but they have been reported to be predacious when plants and seeds are scarce. (Root 1986).

The false chinch bug, Nysius raphanus (Howard) is possibly the most common insect that occurs in the state of High Plains and Southern Rockies. False chinch bugs are found abundantly in hemp throughout the growing season. Winter annual mustards are a common host to this hemipteran and high populations migrate when their host plants are removed due to roadside mowing. The damage that false chinch bugs may produce to hemp is not predicted to affect yield or quality.

Rhopalidae. Harmostes reflexulus (Say) is a scentless plant bug found on many flowering plants as they are known to eat flowers. The genus can be identified by the enlarged metafemora with spines on its legs (Arnett, 2000). This rhopalid was documented from sites that were rich in pollen from flowering hemp although the association of this species with hemp is unclear. Adults are bivoltine and can be commonly encountered on plants in the Asteraceae until late June (Yonke, 1970).

\section{Diptera}

Ulidiidae. Physiphora demandata (Fabricius) are small greenish dipterans found in deciduous woodlands being grazed by cattle and in partially shaded pastures (Allen and Foote, 1967). Larvae are known to develop well on decaying vegetation (Allen and Foote, 1967). This fly has been found occasionally in hemp in Larimer and Weld counties. These saprophagous flies has 
been observed feeding and congregating around exudates on hemp plants. Adults can be encountered around accumulations of decaying vegetation or seen in nature feeding on animal droppings. Hemp fields using manure may come to observe this incidental dipteran.

\section{Effects of hemp cultivar type on arthropod diversity}

In each year of this survey, there was a mixture of hemp types sampled. Most were crops primarily grown for $\mathrm{CBD}(\mathrm{N}=3,10,10$ in 2016-2018, respectively) the remainder being crops grown for seed and/or fiber ( $\mathrm{N}=2,4,5$ in 2016-2018, respectively). This allowed a comparison of the diversity of arthropods detected in fields of these different hemp types.

All years showed differences in arthropod diversity between these two types of hemp crops (Figure 2.4). Summarized diversity index means within a season were consistently lower in CBD hemp versus seed/fiber varieties and these differences were similar in all three years: 2.3 vs. 3.2 in $2016,2.2$ vs. 3.0 in 2017 , and 2.1 vs. 3.4 in 2018.

Perhaps the most obvious difference in the culture of these two crops that could explain differences in diversity would be the presence of male flowers and pollen on seed/fiber crops, which would be largely or completely absent in the CBD crops surveyed. Many insect species detected in surveys (Table 2.4) were pollen feeders, notably bees, various pollen feeding beetles and western flower thrips. Some insects, including predatory species such as lady beetles and minute pirate bugs, are known to sustain themselves on pollen when prey is scarce. Where pollen collects and ferments it may be attractive to various flies or scarab beetles that are attracted to decomposing material. In addition, the male flowers are a source of food observed to be favored by some common herbivores, notably the zebra caterpillar and false chinch bug.

However, differences in diversity between these two hemp types were observed throughout the season, including periods prior to flower/pollen production (Table 2.7). In 2017, 
the average diversity index was 2.3 vs. 3.0 in June, 2.1 vs. 3.2 in July, and 2.3 vs. 3.1 in August, for CBD vs. seed/fiber crops. In 2018, these differences were similar: 2.0 vs. 3.2 in June, 2.2 vs. 3.4 in July, and 2.1 vs. 3.4 in August, for the two crops. Probability values for 2016, 2017, and 2018 were statistically significant to the 0.0001 level. These differences may be largely explained by differences in the production of these two crops. Seed/fiber crops, grown from seed, are planted much earlier in the season, often in May, compared to transplanted CBD crops moved into fields typically in June. This allows for a longer period when insects can establish on a crop. Plant densities are much higher in seed/fiber crops, which can allow for greater plant cover of a field throughout the season. Differences in plant architecture may also contribute to differences in insect colonization, with seed/fiber crops being much taller than CBD crops. Furthermore, the use of plastic mulches, a common practice with CBD crops, was not observed with seed/fiber crops, and this practice may affect insects, particularly those with soil stages. 

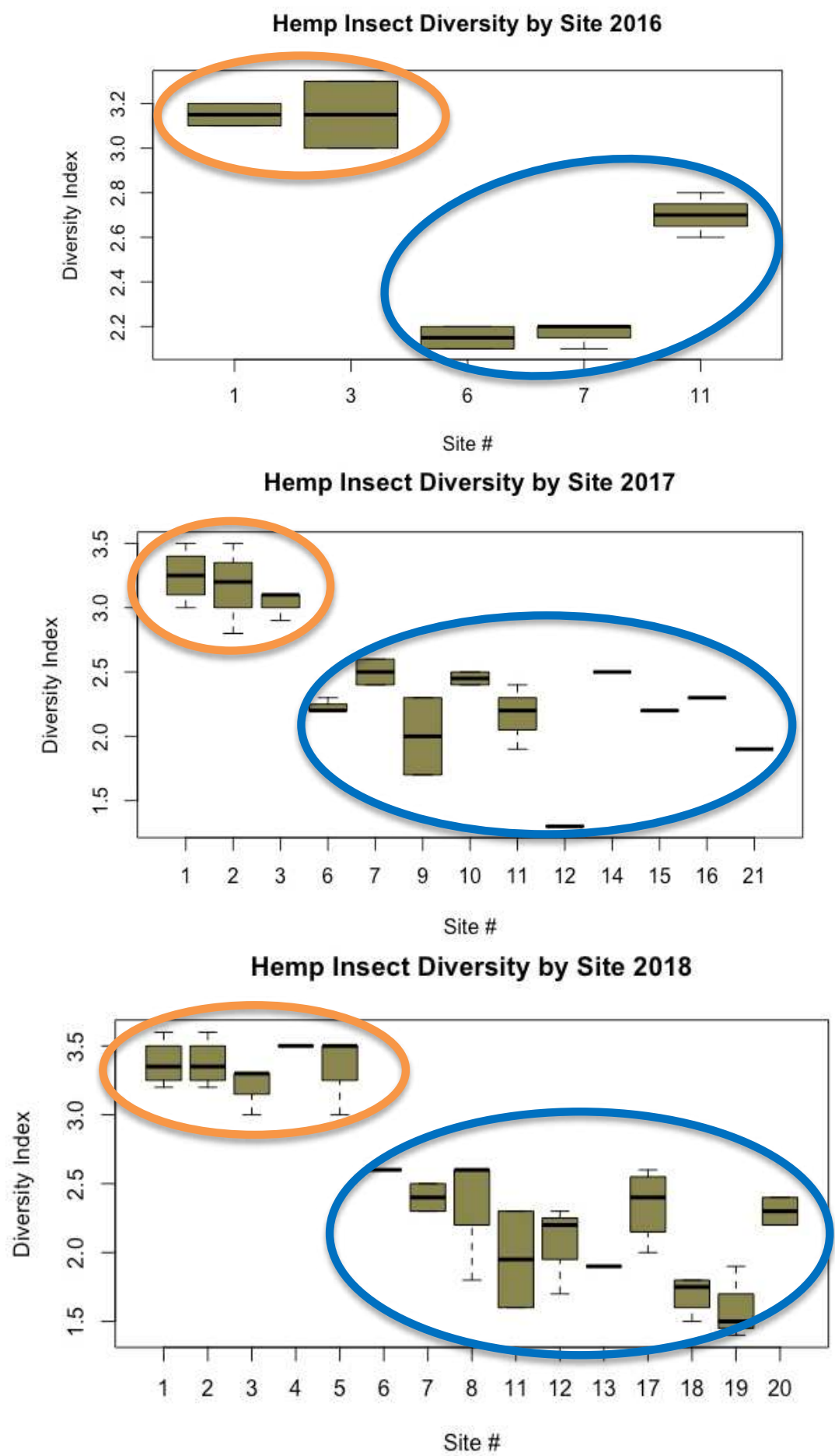

Figure 2.4 Comparison of overall arthropod diversity in CBD versus seed/fiber types of hemp based on sweep net sampling of hemp fields in eastern Colorado over a three year period, 2016-2018. Box plots were calculated using R statistical software. Cultivars produced for seed/fiber/grain are indicated with the color orange and cultivars produced for cannabidiol (CBD) are indicated with the color blue. 
Table 2.7 Relative diversity of hemp insects detected in field surveys in eastern Colorado during 20162018. Means are presented using a Shannon-Wiener Diversity Index $(\mathrm{H})$ calculated in excel by dividing the proportion of each individual in a sample by its natural $\log (\mathrm{P} 1 * \ln \mathrm{P} 1)$. The summation of all $(\mathrm{Pi} * \ln$ $\mathrm{Pi}$ ) products from each species produced $\mathrm{H}$ values, the Shannon-Wiener Diversity Index, representing species richness and abundance for that site. High values of $\mathrm{H}$ are representative of more diverse communities. Mean, standard error, and grouping was produced by generating pairwise comparisons of emmeans through a cld display using R statistical software.

Site \# Hemp Type June July August Sept Mean SE Group 2016 Surveys

$\begin{array}{ll}\text { Site } 1 & \text { Seed/Fiber } \\ \text { Site } 3 & \text { Seed/Fiber } \\ \text { Site } 6 & \text { CBD } \\ \text { Site } 7 & \text { CBD } \\ \text { Site } 11 & \text { CBD }\end{array}$

$\begin{array}{lll}- & 3.1 & 3.2 \\ - & 3 & 3.3 \\ 2.1 & 2.2 & - \\ 2.2 & 2.1 & 2.2 \\ 2.6 & 2.8 & 2.7\end{array}$

$\begin{array}{lll}- & 3.150 & 0.076 \\ - & 3.150 & 0.076 \\ - & 2.150 & 0.076 \\ - & 2.166 & 0.062 \\ - & 2.700 & 0.062\end{array}$

3
3
1
1
2

\section{Surveys}

$\begin{array}{lllllllll}\text { Site } 1 & \text { Seed/Fiber } & 3.3 & 3.5 & 3.2 & - & 3.250 & 0.115 & 5 \\ \text { Site 2 } & \text { Seed/Fiber } & 2.8 & 3.2 & 3.5 & - & 3.166 & 0.132 & 5 \\ \text { Site 3 } & \text { Seed/Fiber } & 3.1 & 3.1 & 2.9 & - & 3.033 & 0.132 & 45 \\ \text { Site 5 } & \text { Seed/Fiber } & 3 & 3 & 2.8 & - & 2.233 & 0.132 & 23 \\ \text { Site 6 } & \text { CBD } & 2.3 & 2.2 & 2.2 & - & 2.233 & 0.132 & 23 \\ \text { Site 7 } & \text { CBD } & - & 2.4 & 2.6 & - & 2.500 & 0.162 & 3 \\ \text { Site 9 } & \text { CBD } & - & 1.7 & 2.3 & - & 2.000 & 0.162 & 2 \\ \text { Site 10 } & \text { CBD } & 2.5 & 2.4 & - & - & 2.450 & 0.162 & 23 \\ \text { Site 11 } & \text { CBD } & 2.2 & 2.4 & 1.9 & - & 2.166 & 0.132 & 23 \\ \text { Site 12 } & \text { CBD } & - & 1.3 & - & - & 1.300 & 0.230 & 1 \\ \text { Site 14 } & \text { CBD } & 2.5 & - & - & - & 2.500 & 0.230 & 234 \\ \text { Site 15 } & \text { CBD } & - & 2.2 & - & - & 2.200 & 0.230 & 23 \\ \text { Site 16 } & \text { CBD } & - & - & 2.3 & - & 2.300 & 0.230 & 23 \\ \text { Site 21 } & \text { CBD } & 1.9 & - & - & - & 1.900 & 0.230 & 123\end{array}$

\section{Surveys}

$\begin{array}{lllllllll}\text { Site } 1 & \text { Seed/Fiber } & 3.3 & 3.4 & 3.6 & 3.2 & 3.375 & 0.124 & 6 \\ \text { Site } 2 & \text { Seed/Fiber } & 3.3 & 3.4 & 3.6 & 3.2 & 3.375 & 0.124 & 6 \\ \text { Site 3 } & \text { Seed/Fiber } & 3.3 & 3.3 & 3 & - & 3.200 & 0.143 & 6 \\ \text { Site 4 } & \text { Seed/Fiber } & - & 3.5 & - & - & 3.500 & 0.248 & 6 \\ \text { Site 5 } & \text { Seed/Fiber } & 3 & 3.5 & 3.5 & - & 3.333 & 0.143 & 6 \\ \text { Site 6 } & \text { CBD } & 2.6 & 2.6 & 2.6 & - & 2.600 & 0.143 & 5 \\ \text { Site 7 } & \text { CBD } & - & 2.3 & 2.5 & - & 2.400 & 0.175 & 345 \\ \text { Site 8 } & \text { CBD } & 1.8 & 2.6 & 2.6 & - & 2.333 & 0.143 & 345 \\ \text { Site 11 } & \text { CBD } & 2.3 & 1.6 & - & - & 1.950 & 0.175 & 1234 \\ \text { Site 12 } & \text { CBD } & 2.3 & 2.2 & 1.7 & - & 2.066 & 0.143 & 234 \\ \text { Site 13 } & \text { CBD } & - & 1.9 & 1.9 & - & 1.900 & 0.175 & 123 \\ \text { Site 17 } & \text { CBD } & 2 & 2.6 & 2.5 & 2.3 & 2.350 & 0.124 & 45 \\ \text { Site 18 } & \text { CBD } & 1.7 & 1.8 & 1.8 & 1.5 & 1.700 & 0.124 & 12 \\ \text { Site 19 } & \text { CBD } & 1.4 & 1.9 & 1.5 & - & 1.600 & 0.143 & 1 \\ \text { Site 20 } & \text { CBD } & - & 2.4 & 2.2 & - & 2.300 & 0.175 & 345\end{array}$




\section{CONCLUSION}

Seventy-three families of arthropod species were collected from hemp and described from eight counties in Colorado in 2016, 2017, and 2018. Emerging pests of the crop that are described are; corn earworm (Helicoverpa zea), hemp russet mite (Aculops cannibicola), cannabis aphid (Phorodon cannabis), and Eurasian hemp borer (Grapholita delineana). Local outbreaks of several species of grasshoppers have been observed to produce significant crop injury, particularly by twostriped grasshopper (Melanoplus bivittatus), as species that frequently chews on stems and may cause girdling and stem breakage. Natural enemies were abundant throughout all Colorado hemp fields and provide robust ecosystem services. As production of this crop continues and expands changes in the arthropod complex can be expected. These baseline observational studies and descriptions of arthropod associations with the crop provide

fundamental information needed to identify and develop arthropod pest management approaches for hemp in Colorado. 


\section{REFERENCES}

Abbott, C. H. 1962. A migration problem-Vanessa cardui (Nymphalidae), the painted lady butterfly. J. Lepidoptera Society, 16: 229-233.

Alexander, G. 1941. Keys for the identification of Colorado Orthoptera. University of Colorado Studies, Series D. 1:129-164.

Allen, E. J. and B.A. Foote. 1967. Biology and immature stages of three species of Otitidae (Diptera) which have saprophagous larvae. Annals of the Entomological Society of America, 60(4), 826-836.

Anonymous., R Core Team. 2019. R: A language and environment for statistical computing. R Foundation for Statistical Computing, Vienna, Austria. version 3.5.3.

URL http://www.R-project.org/

Arnett R.H., Jr. 2000. American insects, a handbook of insects of America north of Mexico. USA, FL-Boca Raton: CRC. 1024pp.

Ball, E. D. et al. 1942. The grasshoppers and other Orthoptera of Arizona. Ariz. Agr. Exp. Sta. Tech. Bull. 93:255-373.

Bailey, C. L. and H. L. Chada. 1968. Spider populations in grain sorghums. Annals of the Entomological Society of America 61: 567-572.

Bishopp, F. C. 1929. The Bollworm or Corn Ear Worm as a Cotton Pest. Washington, D.C.: U.S. Department of Agriculture,. Print. Farmers' Bulletin (United States. Department of Agriculture); No. 1595

Blatchley, W. S. 1920. Orthoptera of Northeastern America. The Nature Publishing Co., Indianapolis, Indiana. 784 pp.

Blatchley, W. S. 1926. Heteroptera or true bugs of Eastern North America, with especial reference to the faunas of Indiana and Florida. Nature Publishing Co. Indianapolis. 1116 pp.

Branson T.F., and J. L. Krysan. 1981. Feeding and oviposition behavior and life cycle strategies of Diabrotica: an evolutionary view with implications for pest management. Environ. Entomol. 10: 826-831. 
Bruner, L. 1897. The grasshoppers that occur in Nebraska. Annu. Rep. Entomol., Neb. State Bd. Agr., 1896, 105-138.

Bunn, Bonnie; D., Alston; and M., Murray. 2015. "Flea Beetles on Vegetables". All Current Publications. Paper 903. URL at https://digitalcommons.usu.edu/extension_curall/903

Capinera, J. L., and T.S. Sechrist. 1982. Grasshoppers (Acrididae) of Colorado: identification, biology and management. Colorado Agricultural Experiment Station; Bulletin 584S.

Chittenden, EH. and Marsh, H.O. 1920. The Western Cabbage Flea Beetle. U.S. Dep. Agric. Tech. Bull. 902. 21pp.

Clark, E. W. and P. A. Glick. 1961. Some predators and scavengers feeding upon pink bollworm moths. J. Econ. Entomol. 54: 815-816.

Cranshaw, W. and D., Shetlar. 2018. Garden insects of North America: The ultimate guide to backyard bugs (Second ed.). Princeton: Princeton University Press. 704pp.

Cranshaw, W. et al. 2018. Phorodon cannabis Passerini (Hemiptera: Aphididae), a newly recognized pest in North America found on industrial hemp. Insecta Mundi 0662: 1-12.

Cranshaw, W. et al. (2019). Developing Insect Pest Management Systems for Hemp in the United States: A Work in Progress. Journal of Integrated Pest Management, 10(1), 26.

Cranshaw, W. S., D.A. Leatherman, and J.R. Feucht. 2009. Leafmining insects. Colroado State University Service-in-Action; no. 5.548.

Cokendolpher, J. C., Horner, N. V., \& Jennings, D. T. 1979. Crab spiders of north-central Texas (Araneae: Philodromidae and Thomisidae). Journal of the Kansas Entomological Society, 723734.

Cook K., and R. Weinzierl. 2004. Factsheet on Corn earworm Helicoverpa zea (Boddie) University of Illinois, Department of Crop Sciences

Cole, F. R., and E. I., Schlinger. 1969. The Flies of Western North America. University of California Press, Berkley and Los Angeles. pp 693.

Deitch, R. 2003. Hemp: American History Revisited: The Plant with a Divided History. Algora Publishing, New York. 244 pp. 
Deitz, L. L., \& Wallace, M. S. (2012). Richness of the Nearctic treehopper fauna (Hemiptera: Aetalionidae and Membracidae). Zootaxa, 3423(1), 1-26.

Demirel, N., \& Cranshaw, W. (2006). Effects of previous leaf injuries to spring canola on western black flea beetle ( Phyllotreta pusilla ) feeding. Phytoparasitica, 34(1), 87-91.

Di Marzo, V. 2008. Targeting the endocannabinoid system: to enhance or reduce? Nature Reviews. Drug Discovery 7: S438-S455.

Drummond, F. A., J. A. Collins, B. Choate, D. Woodman, D. T. Jennings, H. Y. Forsythe, and J. C. Cokendolpher. Harvestman (Opiliones) Fauna Associated With Maine Lowbush Blueberry Fields in the Major Production Areas of Washington and Hancock Counties. Environmental Entomology 39.5 (2010): 1428-440.

Dunn, J. A. (1960). The natural enemies of the lettuce root aphid, Pemphigus bursarius (L.). Bulletin of Entomological Research 51(2): 271-278.

Faegri, K., J. Iverson, P. E. Kaland, and K. Krzywinski. 1989. Textbook of pollen analysis, ed. 4th. Wiley, New York. IV Edition. K. Faegri, P.E. Kaland, K. Krzywinski (eds). Chichester, USA: John Wiley \& Sons.

Fall, H. C. (1912). A review of the North American species of Collops (Col.). Journal of the New York Entomological Society, 20(4), 249-274.

Gavloski, J.E., Ekuere, U., Keddie, A., Dosdall, L., Kott, L. and Good, A.G. (2000) Identification and evaluation of flea beetle (Phyllotreta cruciferae) resistance within Brassicaceae. Can. J. Plant Sci. 80:881- 887.

Gillette, C. P. 1905. The beet webworm, Loxostege sticticalis L., Colo. Agr. Exp. Sta. Bull. 98:112

Gillette, C. P. 1904. Annotated list of Colorado Orthoptera. Colo. Agr. Exp. Sta. Bull. 94:17-67

Gómez-Moya, C. A., M.G. Gondim Jr, G. J., de Moraes, and E.G. de Morais, (2018). Effect of relative humidity on the biology of the predatory mite Amblyseius largoensis (Acari: Phytoseiidae). International journal of acarology, 44(8), 400-411.

Gimmel, M. L. 2013). Genus-level revision of the family Phalacridae (Coleoptera: Cucujoidea). Zootaxa, 3605(1), 1-147. 
Green, J. W. 1961. Revision of the species of Pyropyga (Lampyridae). The Coleopterists' Bulletin, 65-74.

Hantsbarger, W. M. 1979. Grasshoppers in Colorado. Colo. St. Univ. Ext. Serv. Bull. 502A:1 30.

Harris, H. M. 1928. A monographic study of the hemipterous family Nabidae as it occurs in North America. Entomol. Am. (NS) 9:1-98

Hebard, M. 1929. The Orthoptera of Colorado. Proc. Nat. Acad . Sci. Phil. 81 :303-425.

Hill, D. S. (1994). Agricultural entomology. Timber Press, 133 SW 2nd Ave., Suite 450, Portland, OR. 665 pp.

Kagan, M. 1943. The Araneida found on cotton in central Texas. Ann. Entomol. Soc. America 36: $257-258$

Kelton L. A. 1965. Chlamydatus Curtis in North America (Hemiptera: Miridae). Canadian Entomologist 97:1132-1144.

Knight H.H 1923. Guide to the insects of Connecticut. Part IV. The Hemiptera or sucking insects of Connecticut-Family Miridae (Capsidae). State of Connecticut Geological and Natural History Survey, Bulletin 34:422-658.

Knight H.H 1941. The plant bugs, or Miridae of Illinois. Bulletin Illinois Natural History Survey 22:1-234.

Knowlton, G. F. 1943. Nabis altcrnatns feeding ob- servations. Bull. Brooklyn Entomol. Soc. 38: 122,140

Kryachko Z, Ignatenko M, Markin A, Zaets V. 1965 Notes on hemp tortrix. Zashchita Rastenil Verdit. Bolez. 5:51-54

Lago, P.K. and D.F. Stanford. 1989. Phytophagous insects associated with cultivated marijuana (Cannabis sativa L.) in northern Mississippi. Journal of Entomological Science: October 1989, Vol. 24, No. 4, pp. 437-445.

Lehtinen, P. T., and Y. M., Marusik (2008). A redefinition of Misumenops FO PickardCambridge, 1900 (Araneae, Thomisidae) and review of the New World species. Arachnology, 14(4), 173-199. 
Linger, P., J. Müssig, H. Fischer, and J. Kobert. 2002. Industrial hemp (Cannabis sativa L.) growing on heavy metal contaminated soil: fiber quality and phytoremediation potential. Industrial Crops and Products, 16(1), 33-42.

McPartland, J.M. 1996. Cannabis pests. Journal of the International Hemp Association 3(2): 49, $52-55$.

McPartland, J.M. 2002. Epidemiology of the hemp borer, Grapholita delineana Walker (Lepidoptera: Oleuthreutidae), a pest of Cannabis sativa L. Journal of Industrial Hemp 7(1): 2542.

McPartland, J.M., R.C. Clarke, and D.P. Watson. 2000. Chapter 4. Insects and Mites. In Hemp Diseases and Pests: Management and Biological Control - An Advanced Treatise. Edited by McPartland, J.M., R.C. Clarke, and D.P. Watson. CABI Publishing. 251 pp.

McPartland, J.M. and K.W. Hillig. 2003. The Hemp Russet Mite. Journal of Industrial Hemp 8(2): 107-112.

Michelbacher, A.E., W.W. Middlekauff, and O.G. Bacon. 1953. Cucumber beetles attacking melons in northern California. J. Econ. Entomol . 46: 489-494.

Miller, W. E. 1982. Grapholita delineana (Walker), a Eurasian Hemp Moth, Discovered in North America. 1982. Annals of the Entomological Society of America 75 (2): 184-186.

Montford, S., and M. Small (1999). A comparison of the biodiversity friendliness of crops with special reference to hemp (Cannabis sativa L.). Journal of International Hemp Association, 6, 53-63.

Mound, L. A. and G. Kibby (1998) Thysanoptera: An Identification Guide, 2nd ed. CABI Int., Wallingford, pp 70.

Mound, L. (2005). Thysanoptera: Diversity and interactions. Annual Review of Entomology, 50, 247-69.

Musto, D. F. (1991). Opium, cocaine and marijuana in American history. Scientific American, 265(1), 40-47. 
Mulkern, G. B., K. P. Pruess, H. Knutson, A. F. Hagen, J. B. Campbell and J. D. Lambley. 1969. Food habits and preferences of grassland grasshoppers of the north central Great Plains. No. Oak. Agr. Exp. Sta. Bull. 481:1-32.

Müller, F. P., and K. Ewald. (1976). Beitrag zur Kenntnis der Bionomie und Morphologie der Hanfblattlaus Phorodon cannabis Passerini, 1860 (Homoptera: Aphididae). Beiträge zur Entomologie Contributions to Entomology, 26(2), 455-463.

Nartshuk, E. P. (2000): Outbreaks of carnivorous fly Thaumatomyia notata MEIGEN Diptera: Chloropidae) and their periodicity. Zoological Institute, Russian Academy of Sciences, Universitetskaya. Volume 1, St. Petersburg, Russia. 286: 93-100

Newton B.L. Yeargan K.V. 2001. Predation of helicoverpa zea (Lepidoptera: Noctuidae) eggs and first instars by Phalangium opilio (Opiliones: Phalangiidae). J. Kans. Entomol. Soc . 74: 199-204.

Newton, H.C.E (1928) The biology of flea beetles (Phyllotreta) attacking cultivated Cruciferae. Trans. EntomoL Soc. II. 25:90-115.

O'Brien, C. and Arthi H.S., 2019. Bee diversity and abundance on flowers of industrial hemp (Cannabis sativa L.). Biomass and Bioenergy. 122: 331-335.

O'Donnell, J. E., and Schaefer, C. W. 2018. Annotated checklist of the Pentatomidae (Heteroptera) of Connecticut. The Great Lakes Entomologist, 45(3 \& 4), 9.

Olfert, O. 1986. Insect pests of wheat in western Canada and their control. In Wheat Production in Canada-A Review (pp. 415-430). University of Saskatchewan Saskatoon, Canada.

Olfert, O. and A. Slinkard. 1999. Grasshopper (Orthoptera: Acrididae) damage to flowers and pods of lentil (Lens culinaris L.). Crop Protection, 18(8), 527-530.

Ott, R. L., and , M. T. Longnecker. 2010. An introduction to statistical methods and data analysis. Belmont, US Nelson Education. 2: 878-931

Pate, D. W. 1999. Hemp seed: a valuable food source. Advances in hemp research, 243-255.

Peairs, F. B., and J.L. Capinera, 2002. Caterpillars on field crops. III. Insect series. Crops; no. 5.565 . 
Pfadt, R. E. 1994. Field guide to common western grasshoppers. Wyoming Agricultural Experimental Station, Laramie,Wyoming, USA. Second edition. Bulletin number 912.

Pfannenstiel R.S. Yeargan K.V. 2002. Identification and diel activity patterns of predators attacking Helicoverpa zea (Lepidoptera: Noctuidae) eggs in soybean and sweet corn. Environ. Entomol . 31: 232-241.

Pinto-Da-Rocha, R., G. Machado, and G. Giribet. 2007. Harvestmen: The biology of Opiliones. Harvard University Press, February 28, 2007, Cambridge, MA. 17-35.

Pollet, M. A., S.E. Brooks, and J.M. Cumming. 2004. Catalog of the Dolichopodidae (Diptera) of America north of Mexico. Bulletin of the American Museum of Natural History, 2004(283), $1-114$.

Rakhshani, E., et al. 2012. Parasitoids (Hymenoptera: Braconidae: Aphidiinae) of northeastern Iran: Aphidiine-aphid-plant associations, key and description of a new species. Journal of Insect Science, 12(1).

Readio, P. 1924. Notes on the life history of a beneficial reduviid, Sinea diadema (Fabr.), Heteroptera. Journal of Economic Entomology, 17(1), 80-86.

Root, R. B. 1986. The life of a California population of the facultative milkweed bug, Lygaeus kalmii (Heteroptera: Lygaeidae). Proceedings of the Entomological Society of Washington (USA). 88: 201-214.

Sabelis, M. W. and J., Bruin. 1996. Evolutionary ecology: Life history patterns, food plant choice and dispersal in Eriophyoid Mites: Their Biology, Natural Enemies and Control, (Elsevier Science Publishers. 329-365

Sánchez-Ruiz M., F.M ,Fontal-Cazalla, A. Sánchez-Ruiz, and J.I. López-Colón. 1997. El uso de insectos depredadores en el control biológico aplicado. Boletín de la Sociedad Entomológica Aragonesa 20: 141-149.

Sankey J.H.P. and T.H. Savory 1974. British harvestmen Arachnida: Opiliones, keys and notes for the identification of the species. Synopsis of the Bristish Fauna No. 4. The Linnean Society of London, Academic Press, London, pp. 76

Schaefer, C.W. 1991 Catalog of the Heteroptera, or True Bugs, of Canada and the Continental United States, Annals of the Entomological Society of America, 84:1, 132-134 https://doi.org/10.1093/aesa/84.1.132a 
Schuh, R. T. and M.D. Schwartz. 2005. Review of North American Chlamydatus Curtis species, with new synonymy and the description of two new species (Heteroptera: Miridae: Phylinae). American Museum Novitates, 2005(3471), 1-55.

Sell, R.A. 1915. Some notes on the western twelve-spotted and the western striped cucumber beetles. J. Econ. Entomol. 8: 515-520.

Skevington, J. H. and D.K. Yeates. 2001. Phylogenetic classification of Eudorylini (Diptera: Pipunculidae). Systematic Entomology, 26(4), 421-452.

Skevington, J., and S. A. Marshall. 1998. Systematics of New World Pipunculus (Diptera: Pipunculidae). Entomological Society of America, Lanham, Maryland. 201 pp.

Slater, J. A., and R.M. Baranowski. 1978. How to Know the True Bugs (Hemiptera-Heteroptera). William C. Brown Co. Dubuque, Iowa. 256pp.

Slater, J. A. and R. M. Baranowski. 1990. Slater JA, Baranowski RM (1990) The Lygaeidae of Florida (Hemiptera: Heteroptera). Arthropods of Florida and neighboring lands areas. Florida Dep Agric Consumer Serv 14:1-211

Southwood, T. R. and P.A. Henderson. 2009. Ecological methods. John Wiley \& Sons. London 13: $470-484$

Stehr, F. W. 1987. Immature insects. Volume 1. Dubuque, US: Kendall/Hunt Publishing Company. Dubuque, US: 1:288-597

Stehr, F. W. 1991. Immature insects. Volume 2. Dubuque, US: Kendall/Hunt Publishing Company. Dubuque, US: 2:126-144

Trdan, S. et al. 2005. Distribution of Aeolothrips intermedius Bagnall (Thysanoptera: Aeolothripidae) and its potential prey Thysanoptera species on different cultivated host plants. Journal of Pest Science, 78(4), 217-226.

Triplehorn C.A., and N. F. Johnson. 2005. Borror and Delong's Introduction to the study of insects. Seventh edition. Brook/Cole Publishing. Belmont, California. pp 864.

Wheeler, A. G. Jr. 1982. "The Pentatomoidea (Hemiptera) of Northeastern North America with Emphasis on the Fauna of Illinois. J.E. McPherson. Southern Illinois University Press, Carbondale. The Great Lakes Entomologist: Vol. 15: No. 3, Article 17. pp. 240

Available at: http://scholar.valpo.edu/tgle/vol15/iss3/17 
Wheeler, A. G. 2016. Liorhyssus hyalinus (F.)(Hemiptera: Rhopalidae) in the Western United States: New Host Records, Host-Plant Range, and Comments on Use of the Term "Host Plant". Proceedings of the Entomological Society of Washington, 118(1), 115-129.

Whitcomb, W. H., H. Exline, and R. C. Hunter. 1963. Spiders of the Arkansas cotton field. Ann. Entomol. Soc. America 56: 653-660

Yee W.L., P.A. Phillips, J.L. Rodgers, and B.A. Faber 2001 Phenology of arthropod pests and associated natural predators on avocado leaves, fruit, and in leaf litter in Southern California. Environ Entomol 30:892-898

Yonke, T. R. and D.L. D.L. Walker 1970. Field history, parasites, and biology of Harmostes reflexulus (Say) (Hemiptera: Rhopalidae). Journal of the Kansas Entomological Society, 444450.

Young, O. P. 1989a. Interactions between the predators Phidippus audax (Araneae: Salticidae) and Hippodamia convergens (Coleoptera: Coccinellidae) in cotton and in the laboratory. Entomol. News 100:43-47.

Young, O. P. 1989b. Field observations of predation by Phidippus audax (Araneae: Salticidae) on arthropods associated with cotton. J. Entomol. Sci. 24: 266-273. 


\section{CHAPTER 3: THE EFFECT OF INSECTICIDES ON HEMP RUSSET MITES (ACULOPS CANNIBICOLA) INFESTING HEMP (CANNABIS SATIVA)}

Hemp russet mite, Aculops cannibicola (Farkas) (Acari: Eriophyidae), is one of the key pests that have been identified in hemp production (Chapter 2). Indoors, extremely high populations can develop on plants to the point where the mites sometimes completely cover areas of the leaf, becoming visible as a fine powdery material. Outdoors, sustained populations can occur; in one field monitored in Colorado in 2018, russet mite numbers increased steadily from about 50/leaf to about 450/leaf between early June and early September (Chapter 2).

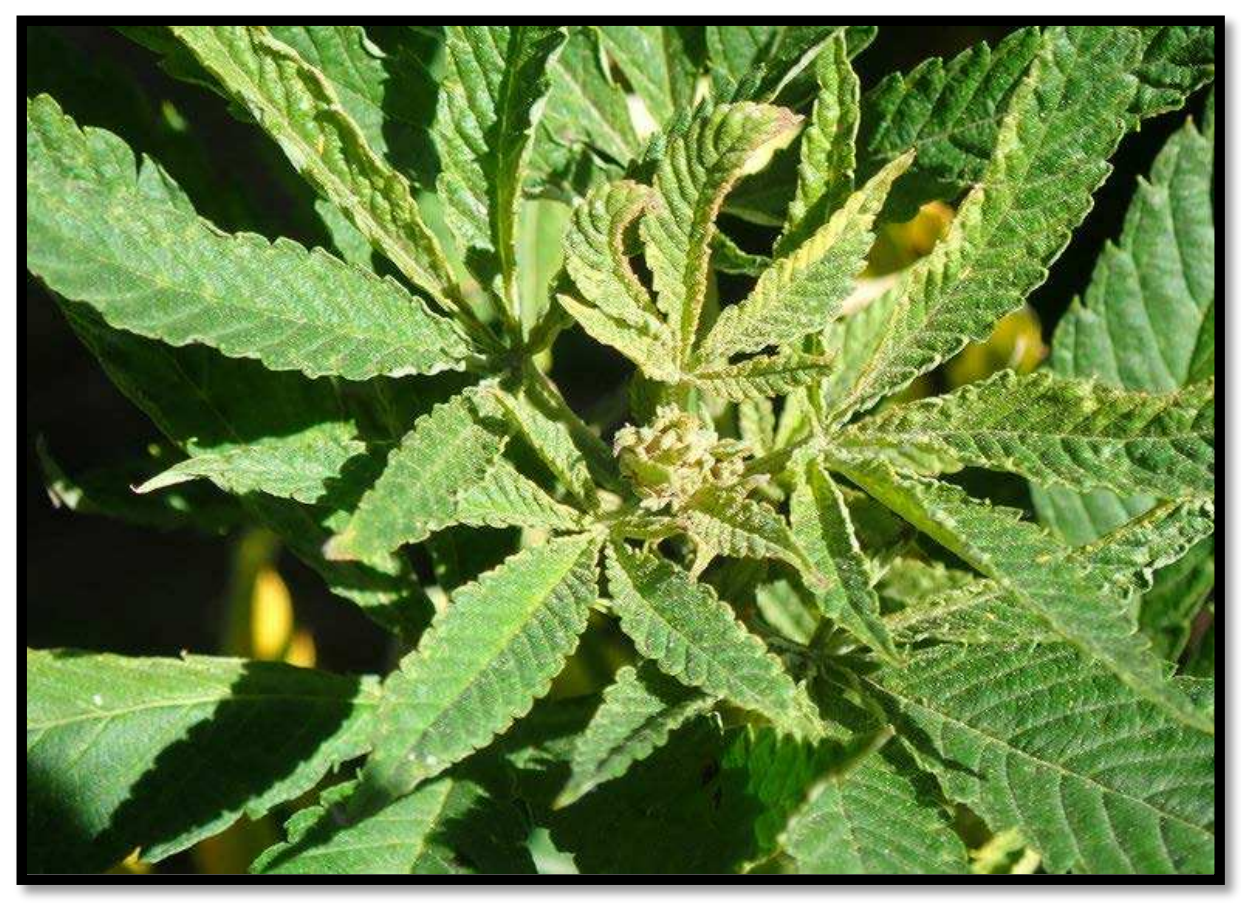

Figure 3.1 Distortions to hemp bud and emerging hemp leaves caused by hemp russet mite.

Leaves that are heavily infested by hemp russet mite often have a slight grayish or bronzed color change due to feeding injuries made to the epidermal cells (Figure 3.1). Leaf size may be reduced and foliage becomes more brittle. In some varieties, a slight upward rolling of the leaf 
edge may occur; this symptom is not universally produced, and some hemp varieties normally will produce similar leaf curling in the absence of the mite. More clearly damaging effects occur when hemp russet mite infests developing flower buds of hemp grown for CBD production. High populations can reduce the size of flower buds and resin production, which can result in reduced yield of extractable cannabinoids (McPartland and Hillig, 2003).

Few, if any, natural enemies attack hemp russet mite and none have been observed to be able to significantly suppress this species either in greenhouses or outdoors in Colorado. Furthermore, no chemical controls have ever been identified for management of this species. To provide better information on possible controls, a test was conducted in 2018 to evaluate products presently allowed for use on hemp for ability to control hemp russet mite.

\section{Methods and materials}

The trial was designed to compare treatments applied as whole plant dips of hemp cuttings for their ability to eliminate hemp russet mite during propagation. The study was conducted indoors in coordination with an established hemp grower in Eaton, Colorado.

Treatments included were:

Suffoil-X (mineral oil)

Dyne-Gro (neem oil)

TetraCURB (rosemary oil)

Green Cleaner (soybean oil/sodium lauryl sulfate)

Water Check

For each treatment, between 45-51 cuttings were taken from mother plants of the same cultivar ('Wife'), known to be infested at the start of the trial (2 August, 2018). Fresh dilutions $(0.5 \%)$ of the test treatments were prepared immediately before treatments were applied. This 
was done by immersing the cuttings into the various treatment mixtures to ensure that the plant (and mites) are coated. The cuttings were then be shaken to remove excess fluid and placed on an absorbent towel. Each cutting was then inserted into plugs for rooting and the cuttings then confined within a clear-top dome to sustain high humidity during the rooting period.

Evaluations were made about 2 weeks following treatments (19 August, 2018). Two evaluations made in this trial of the plants at this time with the first being an evaluation of plant condition on-site. This involved a graduated 0-5 ranking system with plants in excellent condition receiving a 5 and those that were dead a 0 . After ranking the condition of all the plants, from each treatment 10 rooted cuttings were removed to the laboratory at Colorado State University and refrigerated until samples could be processed.

For processing each of the rooted cuttings were cut at the soil line. The mites were then extracted by an alcohol (75\% ethanol) wash. Each plant was then placed in a container, which was then sealed, and gently shaken for about 10 seconds in alcohol to dislodge the mites. The sides of the container were rinsed, and contents were poured into a storage vial, so the dislodged mites could be later counted. Washed plants were discarded.

Because of the large numbers of mites in the samples, a subsample was taken from each vial. This was done by bringing the level of alcohol in the vial to $40 \mathrm{ml}$, then vigorously agitating it to suspend the mites in the solution. At this point a $4 \mathrm{ml}$ subsample was taken from the center of the tube (20-25 $\mathrm{ml}$ mark) and placed in a petri dish to allow the mites to be counted under a dissecting microscope.

All 50 samples (5 treatments x 10 replications) were processed and the mites maintained in alcohol until they could be counted under a dissecting microscope. All processing was done within 48 hours after plants were removed. The mites were subsequently counted under a 
microscope, and a grid placed underneath the dish allowed the contents of the dish to be effectively counted.

Data from both evaluations (mite numbers, plant condition) were analyzed using one-way ANOVA models produced in opensource R statistical software. Treatment means were generated using the emmeans and dpplyr packages in R statistical software (Lenth et al., 2019; Wickman et al., 2018). Once means were produced they were grouped by levels of significance by creating a cld display of pairwise comparisons. Means were then compared for statistical differences in numbers of hemp russet mites found remaining on cuttings two weeks after dipping. After dipping occurred, ranks (0-5) were taken to account for any phytotoxic effects on 15 August 2019 (Table 3.1). Grouped differences among treatment means are shown in (Table 3.1). Significance effects were accepted at $\mathrm{P}<0.05$ for all tests.

\section{Results and discussion}

Small plants can be sensitive to horticultural oils and insecticide treatments. The rooted cuttings were ranked on a scale from 0-5 to determine if there were any phytotoxic effects. TetraCurb dipped plants were statistically different than other treatments and had the lowest ranks (2 out of 5) indicating phytotoxic effects did occur. Suffoil-X and Green Cleaner treated plants showed lower amounts of phytotoxicity as they were not statistically different from the Water Check (Table 3.1).

To confirm which insecticide treatments provided control, mean hemp russet mite numbers were calculated (Table 3.1). Suffoil-X and Green Cleaner were both treatments that showed potential to suppress hemp russet mites. Considering the low levels of mites counted, Suffoil-X and Green Cleaner provide control to hemp russet mites (Table 3.1). 
Horticultural oils are safe to use and pose minimal safety threats to the insecticide

applicator when label language is abided (Johnson, 1985). Advantages of using horticultural oils include safety, effectiveness and limited effects on beneficial insects (Cranshaw and Baxendale, 2005). Important aspects of spraying horticultural oils include: concentration/dose, timing regarding plant phenological development and environmental conditions (Johnson, 1985).

Table 3.1 Mean number of hemp russet mites following five treatment applications are presented. Associated mean plant condition ratings to account for phytotoxic effects of treatments are included. Letters within columns indicate levels of significance. Probability values were all statistically significant to the 0.05 level. Plant condition rating data are distributed normally based on diagnostic plots in R software (QQ plots and Residuals vs. fitted plots).

$\begin{array}{lll} & \text { No. Russet } & \text { Plant Condition } \\ \text { Treatment } & \text { Mites/Plant } & \text { Rating }^{\text {a }}\end{array}$

$\begin{array}{lll}\text { SuffOil-X } & 185.0 \mathrm{a} & 3.88 \mathrm{c} \\ \text { Green Cleaner } & 357.1 \mathrm{a} & 3.48 \mathrm{c} \\ \text { Dyne-Gro } & 1020.7 \mathrm{~b} & 2.86 \mathrm{~b} \\ \text { Water Check } & 1523.2 \mathrm{c} & 3.59 \mathrm{c} \\ \text { TetraCURB } & 1869.0 \mathrm{c} & 2.12 \mathrm{a}\end{array}$

${ }^{\text {a }}$ Plant condition ratings were given on a scale ranging from high phytotoxic effects $(0)$ to no phytotoxic effects (5). A rank of (0) indicated that $100 \%$ of the plant was dead from phytotoxic effects. A rank of (1) indicated $75 \%$ of the plant was chlorotic and growth was stunted. A rank of (2) indicated that $50 \%$ of the plant was chlorotic and growth as stunted. A rank of (3) indicated that $25 \%$ or more of the plant was chlorotic. A rank of (4) indicated $10 \%$ or less of the plant was chlorotic with no stunting of growth. A rank of (5) was given for green, turgid plants. 


\section{REFERENCES}

Anonymous., R Core Team. 2019. R: A language and environment for statistical computing. R Foundation for Statistical Computing, Vienna, Austria. URL http://www.R-project.org/

Cranshaw, W.S., and B. Baxendale. 2005. Insect control: Horticultural oils. Colorado State University Extension Fact Sheet no. 5.569.

Johnson, W. T. 1985. Horticultural oils. Journal of Environmental Horticulture, 3(4), 188-191.

McPartland, J. M., and K.W. Hillig. 2003. The hemp russet mite. Journal of Industrial Hemp, $8(2), 107-112$.

Ott, R. L., and M.T. Longnecker. 2010. An introduction to statistical methods and data analysis. Belmont, US Nelson Education. 2: 878-931

Lenth, R., H. Singmann. J. Love, P. Buerkner, and M. Hervé. 2019. emmeams: Estimated Marginal Means. R package version 1.14.4.

Wickham H., R. François, L. Henry and K. Müller. 2018. dplyr: A Grammar of Data Manipulation. R package version 0.7.6. 


\section{CHAPTER 4: MANAGEMENT RESOURCES FOR HEMP FARMERS PROVIDED BY THE CSU HEMP INSECT WEBSITE}

With hemp becoming a significant crop grown within the United States, producers and potential producers have an enormous number of questions regarding all aspects of its production, including pest management. One small area of need has begun to be addressed through the studies conducted during the projects previously described in this document which have attempted to define the pest management issues associated with arthropods on hemp. Concurrently, to make this newly developed information available for use by producers, various outreach materials have been developed. Most of these are contained within a newly created website, the Colorado State Hemp Insect Website (https://hempinsects.agsci.colostate.edu), launched June 1, 2017.

The purpose of this website is to provide educational resources to hemp producers regarding insect management in industrial hemp. Development of identification materials to provide hemp producers ability to recognize and learn about all arthropods found associated with the crop (in Colorado) was the initial emphasis of this project. This can allow one to distinguish between those species that are potential significant pests from those that pose no serious damage, are incidental to the crop or have beneficial associations (e.g., natural enemies of crop pests). To this end approximately fifty Hemp Insect Fact Sheets have been developed and an insect photo gallery site. Other areas in the website deal with regulatory issues involving insect pest management on the crop and, with a few species, recommendations for management. 


\section{Results and discussion}

In the first 28 months since its establishment (June 1, 2017 to October 3, 2019) there were 19,991 total users that have accessed the Hemp Insect Website (Table 4.1). Eighty percent of users are new to the site and twenty percent are returning users. The Hemp Insect Website has averaged approximately 500 users/week in last 90 days of this period to date, in 2019, there were two thousand monthly users on average. The great majority of visitors, eighty-eight percent $(17,521)$, come from the United States (Google Analytics, October 2019).

Total page views reached 49,284 as of October 3, 2019. The hemp insect home page has $(16 \%)$ of the total page views which means many users are not reaching the full content. The Hemp Insect Fact Sheet page has a significant number of page views (70\%). The Hemp Insect Images page has $(5 \%)$ of total page views accessed. The Got Bugs? page has (3\%) of the total page views. The Regulations and Pesticide Use page has (3.5\%) of the total page views. The Recommendations page has $(3 \%)$ of the total page views. Three minutes is the average length of time that users spend on each website page. 
Table 4.1 Worldwide CSU Hemp Insect Website data (users by region) for the top ten countries. Accessed on October $3^{\text {rd }}, 2019$ using Google Analytics

\begin{tabular}{|c|c|c|c|}
\hline Region & State or Province & Users & Percentage \% \\
\hline & & \multicolumn{2}{|c|}{ Total } \\
\hline & & 19,991 & $\sim 100 \%$ \\
\hline Australia & & 171 & 0.86 \\
\hline \multirow[t]{11}{*}{ Canada } & & 880 & 4.40 \\
\hline & Ontario & 412 & \\
\hline & British Columbia & 148 & \\
\hline & Alberta & 95 & \\
\hline & Quebec & 64 & \\
\hline & Nova Scotia & 52 & \\
\hline & New Brunswick & 39 & \\
\hline & Manitoba & 30 & \\
\hline & Saskatchewan & 27 & \\
\hline & Prince Edward Island & 11 & \\
\hline & Newfoundland and Labrador & 6 & \\
\hline China & & 44 & 0.22 \\
\hline Germany & & 47 & 0.23 \\
\hline India & & 108 & 0.54 \\
\hline Italy & & 157 & 0.78 \\
\hline New Zealand & & 48 & 0.24 \\
\hline South Africa & & 42 & 0.21 \\
\hline United Kingdom & & 204 & 1.02 \\
\hline \multirow[t]{16}{*}{ United States } & & 17,521 & 87.64 \\
\hline & Oregon & 2,777 & \\
\hline & California & 2,106 & \\
\hline & Colorado & 1,986 & \\
\hline & Tennessee & 895 & \\
\hline & Wisconsin & 753 & \\
\hline & Illinois & 733 & \\
\hline & Texas & 645 & \\
\hline & Washington & 645 & \\
\hline & New York & 612 & \\
\hline & North Carolina & 603 & \\
\hline & Michigan & 430 & \\
\hline & Virginia & 430 & \\
\hline & Florida & 398 & \\
\hline & Massachusetts & 356 & \\
\hline & Kentucky & 255 & \\
\hline
\end{tabular}




\section{Conclusion}

Pest management plans, fact sheets, and a photo gallery have been created and made available to the public through the CSU hemp insect website. Goals for the website moving forward involve the creation of new extension materials to aid in integrated pest management. To meet the rising demand for quality content, videos and powerpoints with audio will be developed. Website navigation could be improved by making the drop-down menu on the website more visible. This menu is difficult to locate and many users maybe unaware of its presence. This hidden menu could explain why so many (16\%) of users are viewing the home page. Another issue is that fifty-five percent of users access the website using a mobile device, forty-one percent access it using a desktop, and roughly 6 percent access the website using a tablet. The website was originally designed for use on a laptop or home computer interface, and these data indicate the need for improvements to make this clearly accessible to mobile devices. The results show that this website is meeting a demand, based on use. The CSU hemp insect project has played a central role in providing needed management to researchers, farmers, and the public. 


\section{REFERENCES}

Google Analytics. (2019). CSU Hemp Insect Website Analytics Report. Retrieved from

https://analytics.google.com/analytics/web/\#/reporthome/a24506830w47812802p146739155/\%3

F_u.date $00=20190101 \&$ _u.date $01=20191002$ 